Discrete Comput Geom 27:249-272 (2002)

DOI: $10.1007 / \mathrm{s} 00454-001-0062-7$

\title{
Combinatorics of the Toric Hilbert Scheme
}

\author{
D. Maclagan ${ }^{1}$ and R. R. Thomas ${ }^{2}$ \\ ${ }^{1}$ Department of Mathematics, Stanford University, \\ Stanford, CA 94305, USA \\ maclagan@math.stanford.edu \\ ${ }^{2}$ Department of Mathematics, University of Washington, \\ Seattle, WA 98195, USA \\ thomas@math.washington.edu
}

\begin{abstract}
The toric Hilbert scheme is a parameter space for all ideals with the same multigraded Hilbert function as a given toric ideal. Unlike the classical Hilbert scheme, it is unknown whether toric Hilbert schemes are connected. We construct a graph on all the monomial ideals on the scheme, called the flip graph, and prove that the toric Hilbert scheme is connected if and only if the flip graph is connected. These graphs are used to exhibit curves in $\mathbb{P}^{4}$ whose associated toric Hilbert schemes have arbitrary dimension. We show that the flip graph maps into the Baues graph of all triangulations of the point configuration defining the toric ideal. Inspired by the recent discovery of a disconnected Baues graph, we close with results that suggest the existence of a disconnected flip graph and hence a disconnected toric Hilbert scheme.
\end{abstract}

\section{Introduction}

Let $A=\left[a_{1} \cdots a_{n}\right]$ be a $d \times n$ integer matrix of rank $d$ such that $\operatorname{ker}(A) \cap \mathbb{N}^{n}=\{0\}$ and let $\mathbb{N} A:=\left\{\sum_{i=1}^{n} m_{i} a_{i}: m_{i} \in \mathbb{N}\right\} \subseteq \mathbb{Z}^{d}$ be the non-negative integer span of the columns of $A$. The symbol $\mathbb{N}$ denotes the set of natural numbers including zero. Consider the $\mathbb{N} A$-graded polynomial ring $S:=k\left[x_{1}, \ldots, x_{n}\right]$ over a field $k$ with $\operatorname{deg} x_{i}:=a_{i}$ for all $i$ and an ideal $I \subseteq S$ that is homogeneous with respect to the grading by $\mathbb{N} A$. We say that $I$ is $A$-homogeneous and call the $k$-algebra $R=S / I$ an A-graded algebra if its Hilbert function $H_{R}(b):=\operatorname{dim}_{k}\left(R_{b}\right)=1$ for all $b \in \mathbb{N} A$. Note that if $b$ lies in $\mathbb{Z}^{d} \backslash \mathbb{N} A$, then $R_{b}=\{0\}$. If $S / I$ is an $A$-graded algebra, then $I$ is called an $A$-graded ideal. If $I$ is generated by monomials it is a monomial A-graded ideal.

$A$-graded algebras were introduced by Arnold [1] who investigated matrices of the form $A=\left[\begin{array}{lll}1 & p & q\end{array}\right]$ where $p$ and $q$ are positive integers. A complete classification of all 
$A$-graded algebras arising from $1 \times 3$ matrices can be found in [1], [12], and [13]. The generalization to $d \times n$ matrices is due to Sturmfels [21]. The canonical example of an $A$-graded ideal is the toric ideal of $A$, denoted as $I_{A}$. Initial ideals of $I_{A}$ [22] are also $A$-graded.

In [21] Sturmfels constructed a parameter space whose points are in bijection with the distinct $A$-graded ideals in $S$. This scheme has the same underlying reduced scheme as the toric Hilbert scheme of $A$, denoted as $H_{A}$, which has been defined recently by Peeva and Stillman [14], [15]. The classical Hilbert scheme parameterizes all homogeneous, saturated ideals in $S$ with a fixed Hilbert polynomial, where $S$ is graded by total degree. However, unlike classical Hilbert schemes which are known to be connected [9], it is unknown whether toric Hilbert schemes are connected. Several of the techniques applied to classical Hilbert schemes cannot be used in the toric situation. In particular, the multigraded Hilbert function used to define $A$-graded ideals is not preserved under a change of coordinates. See [15] for further discussions. The only cases in which $H_{A}$ is known to be connected are when $A$ has corank one (i.e., $n-d=1$ ) or two. In the former case the connectivity is trivial, and in the latter it follows from results in [8].

In Section 2 we define a graph on all the monomial $A$-graded ideals in $S$, called the flip graph of $A$, by defining an adjacency relation among these ideals. This generalizes the notion of adjacency between two monomial initial ideals of the toric ideal $I_{A}$, given by the edges of the state polytope of $I_{A}$ [3]. Our main result in Section 3 reduces the connectivity of the toric Hilbert scheme to a combinatorial problem.

Theorem 3.1. The toric Hilbert scheme $H_{A}$ is connected if and only if the flip graph of $A$ is connected.

The flip graph of $A$ provides information on the structure of $H_{A}$. In Section 4 we use these graphs to prove that $2 \times 5$ matrices can have toric Hilbert schemes of arbitrarily high dimension. The projective toric variety of such a matrix is a curve in $\mathbb{P}^{4}$.

Theorem 4.1. For each $j \in \mathbb{N} \backslash\{0\}$, there exists a $2 \times 5$ matrix $A(j)$ such that its toric Hilbert scheme $H_{A(j)}$ has an irreducible component of dimension at least $j$.

In Section 5 we relate the flip graph of $A$ to the Baues graph of $\mathcal{A}$ which is a graph on all the triangulations of the point configuration $\mathcal{A}:=\left\{a_{1}, \ldots, a_{n}\right\} \subset \mathbb{Z}^{d}$ consisting of the columns of $A$. The edges of the Baues graph are given by bistellar flips. This graph and its relatives have been studied extensively in discrete geometry [17]. Sturmfels proved that the radical of a monomial $A$-graded ideal $I$ is the Stanley-Reisner ideal of a triangulation of $\mathcal{A}$, which we denote as $\Delta(\operatorname{rad}(I))$ (see Theorem 4.1 in [21] or Theorem 10.10 in [22]). This gives a map from the vertices of the flip graph into the vertices of the Baues graph. We extend this map to the edges of the flip graph.

Theorem 5.2. If I and $I^{\prime}$ are adjacent monomial A-graded ideals in the flip graph of A, then either they have the same radical and hence $\Delta(\operatorname{rad}(I))=\Delta\left(\operatorname{rad}\left(I^{\prime}\right)\right)$ or $\Delta(\operatorname{rad}(I))$ differs from $\Delta\left(\operatorname{rad}\left(I^{\prime}\right)\right)$ by a bistellar flip. 
In [18] Santos constructed a configuration $\mathcal{A}$ with a disconnected Baues graph, thus settling a central question that remained from the generalized Baues problem (see [17] for a survey) which in its full generality was settled earlier by Rambau and Ziegler [16]. Although Santos's example does not immediately give a disconnected flip graph, it strongly supports the possibility of one. In Section 6 we explain this connection and provide results that point toward a disconnected flip graph and hence, by Theorem 3.1, a disconnected toric Hilbert scheme.

\section{The Flip Graph of $A$}

In this section we define an adjacency relation on all monomial $A$-graded ideals which, in turn, defines the flip graph of $A$. This graph is the main combinatorial object and tool in this paper. We first recall the definition of an $A$-graded ideal.

Definition 2.1. Let $A=\left[a_{1} \cdots a_{n}\right] \in \mathbb{Z}^{d \times n}$ be a matrix of rank $d$ such that $\operatorname{ker}(A) \cap$ $\mathbb{N}^{n}=\{0\}$ and let $\mathbb{N} A:=\left\{\sum_{i=1}^{n} m_{i} a_{i}: m_{i} \in \mathbb{N}\right\}$. An ideal $I$ in $S=k\left[x_{1}, \ldots, x_{n}\right]$ with $\operatorname{deg} x_{i}=a_{i}$ is called an $A$-graded ideal if $I$ is $A$-homogeneous and $R=S / I$ has the $\mathbb{Z}^{d}$-graded Hilbert function:

$$
H_{R}(b):=\operatorname{dim}_{k}\left(R_{b}\right)= \begin{cases}1 & \text { if } b \in \mathbb{N} A \\ 0 & \text { otherwise }\end{cases}
$$

Note that if $b \in \mathbb{Z}^{d} \backslash \mathbb{N} A$, then $R_{b}=\{0\}$ and hence $H_{R}(b)=0$. The canonical example of an $A$-graded ideal is the toric ideal $I_{A}$ which is the kernel of the ring homomorphism $\varphi: S \rightarrow k\left[t_{1}^{ \pm 1}, \ldots, t_{d}^{ \pm 1}\right]$ given by $x_{j} \mapsto t^{a_{j}}$. See [22] for more information. To see that $I_{A}$ is $A$-graded, recall that $I_{A}=\left\langle x^{u}-x^{v}: A u=A v, u, v \in \mathbb{N}^{n}\right\rangle$, and is hence $A$ homogeneous. For each $b \in \mathbb{N} A$, any two monomials $x^{u}$ and $x^{v}$ in $S$ of $A$-degree $b$ (i.e., with $A u=A v=b)$ are $k$-linearly dependent modulo $I_{A}$ making $\operatorname{dim}_{k}\left(\left(S / I_{A}\right)_{b}\right)=1$. If $b \in \mathbb{Z}^{d} \backslash \mathbb{N} A$, then $\left(I_{A}\right)_{b}$ is zero.

Given a weight vector $w \in \mathbb{N}^{n}$, the initial ideal of an ideal $I \subseteq S$ with respect to $w$ is the ideal $\operatorname{in}_{w}(I):=\left\langle\operatorname{in}_{w}(f): f \in I\right\rangle$ where $\operatorname{in}_{w}(f)$ is the sum of all terms in $f$ of maximal $w$-weight. Our assumption that $\operatorname{ker}(A) \cap \mathbb{N}^{n}=\{0\}$ implies that there is a strictly positive integer vector $w^{\prime}$ in the row space of $A$. Using the binomial description of $I_{A}$ given above, we then see that $I_{A}$ is homogeneous with respect to the grading $\operatorname{deg}\left(x_{i}\right)=w_{i}^{\prime}$. Hence, the Gröbner fan of $I_{A}$ covers $\mathbb{R}^{n}$ and each cell in this fan contains a non-zero non-negative integer vector in its relative interior (see Proposition 1.12 in [22]). Therefore, for any weight vector $w \in \mathbb{Z}^{n}$, the initial ideal $\operatorname{in}_{w}\left(I_{A}\right)$ is well defined as it coincides with $\operatorname{in}_{\bar{w}}\left(I_{A}\right)$ where $\bar{w}$ is a non-negative integer vector in the relative interior of the Gröbner cone of $w$. Since the Hilbert function is preserved when passing from an ideal to one of its initial ideals, all initial ideals of $I_{A}$ are also $A$-graded.

If $M$ is a monomial $A$-graded ideal, then for each $b \in \mathbb{N} A$ there is a unique monomial of degree $b$ that does not lie in $M$ and is hence a standard monomial of $M$. Definition 2.1 implies that all $A$-graded ideals are generated by $A$-homogeneous binomials (polynomials with at most two terms) since any two monomials of the same $A$-degree have to be $k$-linearly dependent modulo an $A$-graded ideal. 
There is a natural action of the algebraic torus $\left(k^{*}\right)^{n}$ on $S$ given by $\lambda \cdot x_{i}=\lambda_{i} x_{i}$ for $\lambda \in\left(k^{*}\right)^{n}$.

Definition 2.2. An A-graded ideal is said to be coherent if it is of the form $\lambda \cdot \operatorname{in}_{w}\left(I_{A}\right)$ for some $\lambda \in\left(k^{*}\right)^{n}$ and $w \in \mathbb{Z}^{n}$.

We recall the definition of the Graver basis of $A$ [22]. For $u, v \in \mathbb{N}^{n}$ we write $u<v$ if for each $i=1, \ldots, n, u_{i} \leq v_{i}$ and $u \neq v$.

Definition 2.3. A binomial $x^{u}-x^{v}$ with $A u=A v$ is a Graver binomial if there do not exist $u^{\prime}, v^{\prime} \in \mathbb{N}^{n}$ with $A u^{\prime}=A v^{\prime}$ and $u^{\prime}<u, v^{\prime}<v$. The collection of all Graver binomials is called the Graver basis, $G_{A}$.

The following lemma is a strengthening of Lemma 10.5 in [22] and was also independently discovered by Peeva and Stillman [15]. By the universal Gröbner basis of an ideal we always mean the union of all the finitely many reduced Gröbner bases of the ideal.

Lemma 2.4. Let $I$ be an A-graded ideal, and let $\mathcal{G}=\left\{x^{\alpha_{1}}-c_{1} x^{\beta_{1}}, \ldots\right.$, $\left.x^{\alpha_{k}}-c_{k} x^{\beta_{k}}\right\}$ be the universal Gröbner basis of I. Here the $c_{i}$ may be zero and for each binomial, $x^{\alpha_{i}}$ and $x^{\beta_{i}}$ are not both in I. If $c_{i}=0$, choose $\beta_{i}$ so that $A \alpha_{i}=A \beta_{i}$ and $x^{\beta_{i}} \notin I$. Then for all $i, x^{\alpha_{i}}-x^{\beta_{i}}$ is a Graver binomial. Hence, every minimal generator of $I$ is of this form.

Proof. If $x^{\alpha_{i}}-c_{i} x^{\beta_{i}} \in \mathcal{G}$, then there is some term order $\prec$ such that one of $x^{\alpha_{i}}$ and $x^{\beta_{i}}$ is a minimal generator of $\operatorname{in}_{\prec}(I)$, and the other is standard for $\operatorname{in}_{\prec}(I)$. Since in in $_{\prec}(I)$ is also $A$-graded, it suffices to prove the lemma for monomial $A$-graded ideals, where $c_{i}=0$ for all $i$.

Suppose there exists an $i$ such that $x^{\alpha_{i}}-x^{\beta_{i}}$ is not a Graver binomial. Then there exists $u, v \in \mathbb{N}^{n}$ with $A u=A v$ such that $u<\alpha_{i}$ and $v<\beta_{i}$. Since $I$ is $A$-graded, one of $x^{u}$ or $x^{v}$ is in $I$. If we have $x^{u} \in I$, then $x^{\alpha_{i}}$ would not be a minimal generator of $I$, and if $x^{v} \in I$, then $x^{\beta_{i}}$ would not be standard. Therefore, $x^{\alpha_{i}}-x^{\beta_{i}}$ is a Graver binomial for all $i$.

Definition 2.5. An $A$-homogeneous ideal $I$ in $S$ is weakly A-graded if $H_{S / I}(b) \in\{0,1\}$ for all $b \in \mathbb{N} A$.

Lemma 2.6. Let I be an ideal which for every Graver binomial $x^{\alpha}-x^{\beta}$ contains either a binomial of the form $x^{\alpha}-c x^{\beta}$, where $c$ may be zero, or the monomial $x^{\beta}$. Then I is weakly A-graded.

Proof. It suffices to prove that $M=\operatorname{in}_{\prec}(I)$ is weakly $A$-graded, where $\prec$ is any term order, since in in $_{\prec}(I)$ has the same Hilbert function as $I$. If $x^{\alpha}-x^{\beta}$ is a Graver binomial, then since there is some $c$ with $x^{\alpha}-c x^{\beta} \in I$, one of $x^{\alpha}$ and $x^{\beta}$ lies in $M$. Let $x^{u}$ and $x^{v}$ be two monomials of $A$-degree $b$, and let $x^{\alpha}-x^{\beta}$ be a Graver binomial with $x^{\alpha} \mid x^{u}$ 
and $x^{\beta} \mid x^{v}$. Since one of $x^{\alpha}$ and $x^{\beta}$ lies in $M$, one of $x^{u}$ and $x^{v}$ lies in $M$. It thus follows that there is at most one standard monomial of $M$ in each degree $b$, and so $M$ is weakly $A$-graded.

We now define a "flipping" procedure on a monomial $A$-graded ideal which transforms this ideal into an "adjacent" monomial $A$-graded ideal. The idea is motivated by a similar procedure for toric initial ideals which we describe briefly.

The distinct monomial initial ideals of $I_{A}$ are in bijection with the vertices of the state polytope of $I_{A}$, an $(n-d)$-dimensional polytope in $\mathbb{R}^{n}$ [3]. Two initial ideals are said to be adjacent if they are indexed by adjacent vertices of the state polytope. The edges of the state polytope are labeled by the binomials in the universal Gröbner basis of $I_{A}$, $U G B_{A} \subseteq G r_{A}$.

Suppose $I$ and $I^{\prime}$ are two adjacent monomial initial ideals of $I_{A}$ connected by the edge $x^{\alpha}-x^{\beta}$. The closure of the inner normal cone at the vertex $I$ (respectively $I^{\prime}$ ) is the Gröbner cone $K$ (respectively $K^{\prime}$ ) of $I$ (respectively $I^{\prime}$ ), the interior of which contains all the weight vectors $w$ such that $\operatorname{in}_{w}\left(I_{A}\right)=I$ (respectively $\left.\operatorname{in}_{w}\left(I_{A}\right)=I^{\prime}\right)$. The linear span of the common facet of $K$ and $K^{\prime}$ is the hyperplane $\left\{u \in \mathbb{R}^{n}:(\alpha-\beta) \cdot u=0\right\}$. When $w$ is in the interior of $K, \operatorname{in}_{w}\left(x^{\alpha}-x^{\beta}\right)=x^{\alpha}, x^{\alpha}$ is a minimal generator of $I$ and $x^{\beta} \notin I$. When $w$ is in the interior of $K^{\prime}, \operatorname{in}_{w}\left(x^{\alpha}-x^{\beta}\right)=x^{\beta}, x^{\beta}$ is a minimal generator of $I^{\prime}$ and $x^{\alpha} \notin I^{\prime}$. For a $w$ in the relative interior of the common facet of $K$ and $K^{\prime}$, $\operatorname{in}_{w}\left(x^{\alpha}-x^{\beta}\right)=x^{\alpha}-x^{\beta}$. Hence passing from $I$ to $I^{\prime}$ involves "flipping" the orientation of the binomial $x^{\alpha}-x^{\beta}$. No other binomial in $U G B_{A}$ changes orientation during this passage. See [11] for details. We extend this notion of "flip" to all monomial $A$-graded ideals. Two monomial $A$-graded ideals $M$ and $M^{\prime}$ differ by a "flip" in this more general sense, if there is exactly one binomial $x^{\alpha}-x^{\beta}$ in $G r_{A}$ that changes orientation during the flip.

Definition 2.7. Let $I$ be a monomial $A$-graded ideal and let $x^{\alpha}-x^{\beta}$ be a Graver binomial with $x^{\alpha}$ a minimal generator of $I$ and $x^{\beta} \notin I$. We define $I_{\text {flip }}$, the result of flipping over this binomial, to be

$$
I_{\text {flip }}:=\left\langle x^{\gamma} \mid \exists \delta: x^{\gamma}-x^{\delta} \in G r_{A}, x^{\gamma} \in I, x^{\delta} \notin I, \gamma \neq \alpha\right\rangle+\left\langle x^{\beta}\right\rangle .
$$

Lemma 2.8. The ideal $I_{\text {flip }}$ is weakly A-graded.

Proof. Let $x^{\alpha^{\prime}}-x^{\beta^{\prime}}$ be a Graver binomial, with $x^{\alpha^{\prime}} \in I$. By Lemma 2.6 it suffices to show that either $x^{\alpha^{\prime}} \in I_{\text {flip }}$ or $x^{\beta^{\prime}} \in I_{\text {flip }}$. Since $x^{\alpha^{\prime}} \in I$, there is some Graver binomial $x^{\alpha^{\prime \prime}}-x^{\beta^{\prime \prime}}$ with $x^{\alpha^{\prime \prime}}$ a minimal generator of $I$, and $x^{\beta^{\prime \prime}} \notin I$, and $x^{\alpha^{\prime \prime}} \mid x^{\alpha^{\prime}}$. It is possible that $\alpha^{\prime \prime}=\alpha^{\prime}$ and $\beta^{\prime \prime}=\beta^{\prime}$. If $\alpha^{\prime \prime} \neq \alpha$, then $x^{\alpha^{\prime \prime}} \in I_{\text {flip }}$, and so $x^{\alpha^{\prime}} \in I_{\text {flip. }}$. If $\alpha^{\prime \prime}=\alpha$, then $\beta^{\prime \prime}=\beta$. We divide this situation into three cases:

Case 1: $x^{\beta^{\prime}} \notin I$ and $\alpha^{\prime}=\alpha^{\prime \prime}(=\alpha)$. In this case $\beta^{\prime}=\beta$ and hence $x^{\beta^{\prime}}=x^{\beta} \in I_{\text {flip }}$.

Case 2: $x^{\beta^{\prime}} \notin I$ and $\alpha^{\prime} \neq \alpha^{\prime \prime}(=\alpha)$. In this case $x^{\alpha^{\prime}} \in I_{\text {flip }}$ by definition.

Case 3: $x^{\beta^{\prime}} \in I$. Since $x^{\alpha^{\prime \prime}}=x^{\alpha}$ divides $x^{\alpha^{\prime}}$, and $x^{\alpha^{\prime}}$ and $x^{\beta^{\prime}}$ have disjoint supports, there must be a minimal generator of $I$ different from $x^{\alpha}$ that divides $x^{\beta^{\prime}}$. Since this minimal generator is in $I_{\text {flip }}$ by definition, $x^{\beta^{\prime}} \in I_{\text {flip }}$. 
As defined above, to construct $I_{\text {flip }}$ requires knowledge of the entire Graver basis. However, the local change algorithm in [11] can be used to construct $I_{\text {flip }}$.

Lemma 2.9. The ideal $I_{\text {flip }}$ is the initial ideal with respect to $x^{\alpha} \prec x^{\beta}$ of $W_{\alpha-\beta}=\left\langle x^{\gamma}\right|$ $\gamma \neq \alpha, x^{\gamma}$ is a minimal generator of $\left.I\right\rangle+\left\langle x^{\beta}-x^{\alpha}\right\rangle$.

We note first that this initial ideal is well defined. The only non-trivial $S$-pairs formed during its construction are those of a monomial with $x^{\beta}-x^{\alpha}$, in which case the result is a monomial multiple of $x^{\alpha}$, so there is never any question of what the leading term of a polynomial is. This means that $I_{\text {flip }}$ is in fact the initial ideal of $W_{\alpha-\beta}$ with respect to any term order in which $x^{\alpha} \prec x^{\beta}$. We call $W_{\alpha-\beta}$ a wall ideal since in the coherent situation it is the initial ideal of any weight vector in the relative interior of the common facet/wall between the Gröbner cones of $I$ and $I_{\text {flip }}$ [11].

Proof. Let $K$ be the initial ideal of $W_{\alpha-\beta}$ with respect to $x^{\alpha} \prec x^{\beta}$. We first show the containment $K \subseteq I_{\text {flip }}$. Let $x^{\gamma}$ be a minimal generator of $K$. If $x^{\gamma}=x^{\beta}$, or $x^{\gamma}$ is a minimal generator of $I$ other than $x^{\alpha}$, then $x^{\gamma} \in I_{\text {flip }}$. So we need only consider the case that $\gamma=r \alpha+g$, where $r>0$ and $\alpha, \beta \not \leq g$, as this is the only other form minimal generators of $K$ can have. In order to show that $x^{\gamma}$ is in $I_{\text {flip }}$, it suffices to show that $x^{\gamma}-x^{\delta}$ is a Graver binomial, where $x^{\delta}$ is the unique standard monomial of $I$ of the same $A$-degree as $x^{\gamma}$.

Suppose $x^{\gamma}-x^{\delta}$ is not a Graver binomial, so we can write $\gamma=\sum u_{i}+g^{\prime}, \delta=$ $\sum v_{i}+g^{\prime}$, where for each $i, x^{u_{i}}-x^{v_{i}}$ is a Graver binomial. Since $x^{\delta} \notin I$, we must have $x^{u_{i}} \in I$ and $x^{v_{i}} \notin I$ for all $i$. If $u_{i} \neq \alpha$ for some $i$, this would mean that $x^{u_{i}}$, and hence $x^{\gamma}$, was in $I_{\text {flip }}$. We can thus reduce to the case where $g^{\prime}=g$ and $v_{i}=\beta$ for all $i$, and so $\delta=r \beta+g$. Now since $x^{\gamma}$ is a minimal generator of $K$, there must be a chain of $S$-pairs and reductions leading to $x^{\gamma}$. The chain must start with some minimal generator $x^{\alpha_{1}} \neq x^{\alpha}$ of $I$, and continue to $x^{\alpha_{2}}$, which is the result of reduction by $x^{\beta}-x^{\alpha}$ of $S\left(x^{\alpha_{1}}, x^{\beta}-x^{\alpha}\right)$. The chain continues, with $x^{\alpha_{i}}$ being the result of the reduction by $x^{\beta}-x^{\alpha}$ of $S\left(x^{\alpha_{i-1}}, x^{\beta}-x^{\alpha}\right)$, until reaching $x^{\alpha_{k}}=x^{\gamma}$. We observe that $x^{\alpha_{i}}=x^{r_{i} \alpha+g_{i}}$, for all $2 \leq i \leq k$, with $r_{i} \in \mathbb{N}, r_{k}=r$, and $g_{i} \in \mathbb{N}^{n}$. Since $x^{\alpha}$ and $x^{\beta}$ have disjoint supports, the $S$-pair of $x^{\alpha_{i-1}}=x^{r_{i-1} \alpha+g_{i-1}}$ and $x^{\beta}-x^{\alpha}$ is the monomial $\left[\operatorname{lcm}\left(x^{g_{i-1}}, x^{\beta}\right) / x^{\beta}\right] x^{r_{i-1} \alpha} x^{\alpha}$. This monomial is reduced to $x^{\alpha_{i}}=x^{r_{i} \alpha+g_{i}}$ by $x^{\beta}-x^{\alpha}$. Hence $r_{i} \geq r_{i-1}+1>r_{i-1}$. The monomial $x^{g_{i}}$ is the remainder of $\operatorname{lcm}\left(x^{g_{i-1}}, x^{\beta}\right) / x^{\beta}$ after reduction by $x^{\beta}-x^{\alpha}$. Since $\operatorname{lcm}\left(x^{g_{i-1}}, x^{\beta}\right) / x^{\beta}$ divides $x^{g_{i-1}}$, we see that $x^{g_{i}} \operatorname{divides} x^{g_{i-1}}$. The monomial $x^{g_{i-1}-g_{i}}$ got reduced to zero by $x^{\beta}-x^{\alpha}$ and hence $g_{i-1}-g_{i} \leq\left(r_{i}-r_{i-1}\right) \beta$. Repeating this argument, we see that if $i<j$, then $0<r_{i}<r_{j}, x^{g_{j}} \mid x^{g_{i}}$, and $g_{i}-g_{j} \leq$ $\left(r_{j}-r_{i}\right) \beta$. The last fact implies that $x^{r_{i} \beta+g_{i}}$ divides $x^{r_{j} \beta+g_{j}}$. Further, since $x^{\alpha_{2}}=x^{r_{2} \alpha+g_{2}}$ is the result of reducing $S\left(x^{\alpha_{1}}, x^{\beta}-x^{\alpha}\right)=\left(\operatorname{lcm}\left(x^{\alpha_{1}}, x^{\beta}\right) / x^{\beta}\right) x^{\alpha}$ by $x^{\beta}-x^{\alpha}$, we get $S\left(x^{\alpha_{1}}, x^{\beta}-x^{\alpha}\right)=x^{r^{\prime} \alpha+r^{\prime \prime} \beta+g_{2}}$ where $r^{\prime}+r^{\prime \prime}=r_{2}$ and $\operatorname{lcm}\left(x^{\alpha_{1}}, x^{\beta}\right)=x^{\left(r^{\prime}-1\right) \alpha+\left(r^{\prime \prime}+1\right) \beta+g_{2}}$. However, since $\alpha_{1} \neq \alpha$ and $\alpha$ and $\beta$ have disjoint supports, we must have $r^{\prime}-1=0$ and $r^{\prime \prime}+1=r_{2}$. Hence $x^{\alpha_{1}}$ divides $x^{r_{2} \beta+g_{2}}$. Combining this with the fact that $x^{r_{i} \beta+g_{i}}$ divides $x^{r_{j} \beta+g_{j}}$ whenever $i<j$ and we can conclude that $x^{\alpha_{1}}$ divides $x^{r \beta+g}=x^{\delta}$. This implies that $x^{\delta} \in I$, a contradiction. Therefore, this case cannot occur and we conclude that $K \subseteq I_{\text {flip }}$. 
We now show the reverse inclusion. Suppose $x^{\gamma}$ is a minimal generator of $I_{\text {flip }}$ not equal to $x^{\beta}$, and $x^{\gamma}-x^{\delta}$ is the corresponding Graver binomial with $x^{\delta} \notin I$. We may assume that $x^{\gamma}$ is a multiple of $x^{\alpha}$, as otherwise it lies in $W_{\alpha-\beta}$, and thus is in $K$ automatically. Write $\gamma=r \alpha+\gamma^{\prime}$, where $\alpha \not \leq \gamma^{\prime}$. Suppose that $x^{r \beta+\gamma^{\prime}} \notin I$. Then $\delta=r \beta+\gamma^{\prime}$, so we must have $\gamma^{\prime}=0$ and $r=1$ to preserve $x^{\gamma}-x^{\delta}$ being a Graver binomial. However, then $\gamma=\alpha$, contradicting $x^{\gamma}$ being a minimal generator of $I_{\text {flip }}$. Thus $x^{r \beta+\gamma^{\prime}} \in I$, and so there is some $\alpha^{\prime} \neq \alpha$ with $x^{\alpha^{\prime}}$ a minimal generator of $I$ such that $\alpha^{\prime} \leq r \beta+\gamma^{\prime}$. This means that $x^{r \beta+\gamma^{\prime}} \in W_{\alpha-\beta}$, and so $x^{r \alpha+\gamma^{\prime}}=x^{\gamma} \in W_{\alpha-\beta}$ because $x^{\beta}-x^{\alpha} \in W_{\alpha-\beta}$. Any monomial in $W_{\alpha-\beta}$ is in $K$, so we conclude that $x^{\gamma} \in K$.

Definition 2.10. We say that a binomial $x^{\alpha}-x^{\beta}$ in the Graver basis is flippable for a monomial $A$-graded ideal $I$ if $x^{\alpha}$ is a minimal generator of $I, x^{\beta} \notin I$, and the ideal $I_{\text {flip }}$ obtained by flipping $I$ over $x^{\alpha}-x^{\beta}$ is again a monomial $A$-graded ideal.

We now give a characterization of when a binomial is flippable.

Theorem 2.11. Let I be a monomial A-graded ideal, and let $x^{\alpha}-x^{\beta}$ be a Graver binomial. Then $x^{\alpha}-x^{\beta}$ is flippable for I if and only if I is the initial ideal with respect to $x^{\beta} \prec x^{\alpha}$ of the wall ideal $W_{\alpha-\beta}=\left\langle x^{\gamma}\right| \gamma \neq \alpha, x^{\gamma}$ is a minimal generator of $I\rangle+\left\langle x^{\alpha}-x^{\beta}\right\rangle$.

Proof. Since $W_{\alpha-\beta}$ is $A$-homogeneous, $I$ is the initial ideal of $W_{\alpha-\beta}$ if and only if $W_{\alpha-\beta}$ is an $A$-graded ideal. However, by Lemma $2.9 I_{\text {flip }}$ is an initial ideal of $W_{\alpha-\beta}$, so is $A$-graded exactly when $W_{\alpha-\beta}$ is.

Definition 2.12. The flip graph of $A$ has as its vertices all the monomial $A$-graded ideals in $S$. There is an edge labeled by the Graver binomial $x^{\alpha}-x^{\beta}$ between two vertices $I$ and $I^{\prime}$, if $I^{\prime}$ can be obtained from $I$ by flipping over $x^{\alpha}-x^{\beta}$.

Remark 2.13. The edge graph of the state polytope of $I_{A}$ is a subgraph of the flip graph of $A$. Since the state polytope of $I_{A}$ is $(n-d)$-dimensional, this subgraph is $(n-d)$-connected and so every vertex in this subgraph has valency at least $n-d$.

Let Flips $_{A}$ denote the set of binomials labeling the edges of the flip graph of $A$. Since the edges of the state polytope of $I_{A}$ are labeled by the elements in $U G B_{A}$, we have $U G B_{A} \subseteq$ Flips $_{A} \subseteq G r_{A}$.

Remark 2.14. (i) Gasharov and Peeva [8] proved that all monomial $A$-graded ideals of corank two matrices are coherent. Hence, in this case, the flip graph of $A$ is precisely the edge graph of the state polytope of $I_{A}$, which is a polygon since $n-d=2$, and $U G B_{A}=$ Flips $_{A}$. However, even in this case, Flips A $_{A}$ may be properly contained in $G r_{A}$ : for $A=\left[\begin{array}{lll}1 & 3 & 7\end{array}\right], U G B_{A}=$ Flips $_{A}=\left\{a^{2} c-b^{3}, a^{3}-b, a c^{2}-b^{5}, b^{7}-c^{3}, c-a^{7}, a b^{2}-c\right\}$ while $G r_{A}=$ Flips $_{A} \cup\left\{a^{4} b-c\right\}$.

(ii) For $A=\left[\begin{array}{lll}1 & 3 & 4\end{array}\right], U G B_{A}=$ Flips $_{A}=G r_{A}=\left\{a c^{2}-b^{3}, a^{2} c-b^{2}, b^{4}-c^{3}, b-\right.$ $\left.a^{3}, a b-c, a^{4}-c\right\}$. 


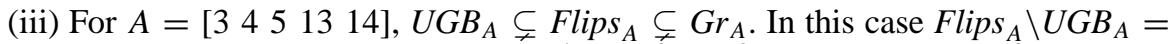
$\left\{a^{2} b c d-e^{2}\right\}$ while $G r_{A} \backslash$ Flips $_{A}=\left\{d^{4}-b c^{4} e^{2}, a d^{3}-b c^{2} e^{2}, e^{3}-b^{6} c d, b^{3} c d^{3}-e^{4}, e^{3}-\right.$ $\left.a^{2} c^{2} d^{2}, e^{2}-a b^{5} c, e^{3}-a b^{2} c d^{2}, e^{3}-a^{4} b d^{2}\right\}$.

For fixed $A$, let $S_{A}$ be the intersection of all the monomial $A$-graded ideals in $S$ and let $P_{A}:=\left\langle x^{\alpha} x^{\beta}: x^{\alpha}-x^{\beta} \in G r_{A}\right\rangle$. Then $P_{A}$ is contained, sometimes strictly, in $S_{A}$ since for each Graver binomial $x^{\alpha}-x^{\beta}$, at least one of $x^{\alpha}$ or $x^{\beta}$ belongs to each monomial $A$-graded ideal.

Lemma 2.15. If $x^{\alpha}-x^{\beta} \in G r_{A}$ has at least one of $x^{\alpha}$ or $x^{\beta}$ in $P_{A}$, then $x^{\alpha}-x^{\beta} \in$ $\mathrm{Gr}_{A} \backslash$ Flips $_{A}$. The converse is false.

Proof. Suppose $x^{\alpha}-x^{\beta}$ is a flippable binomial for a monomial $A$-graded ideal $I$ such that $x^{\alpha} \in I$ and $x^{\beta} \notin I$. If $x^{\alpha} \in P_{A} \subseteq S_{A}$, then $x^{\alpha} \in I_{\text {flip }}$ and if $x^{\beta} \in P_{A} \subseteq S_{A}$, then $x^{\beta} \in I$ both of which are contradictions. To see that the converse is false, consider

$$
A=\left(\begin{array}{llllll}
2 & 1 & 0 & 1 & 0 & 0 \\
0 & 1 & 2 & 0 & 1 & 0 \\
0 & 0 & 0 & 1 & 1 & 2
\end{array}\right)
$$

which has 29 monomial $A$-graded ideals, all of which are coherent. The binomial $x_{1} x_{4} x_{6}-$ $x_{2} x_{3} x_{5} \in G r_{A} \backslash F$ Flips $_{A}$, but neither $x_{1} x_{4} x_{6}$ nor $x_{2} x_{3} x_{5}$ lies in $P_{A}=\left\langle x_{1} x_{2}^{2} x_{4}, x_{1} x_{3}^{2} x_{6}\right.$, $\left.x_{4} x_{5}^{2} x_{6}, x_{1} x_{2} x_{3} x_{5}, x_{2} x_{3} x_{4} x_{5}, x_{2} x_{3} x_{5} x_{6}, x_{1} x_{2}^{2} x_{5}^{2} x_{6}, x_{2}^{2} x_{3}^{2} x_{4} x_{6}, x_{1} x_{3}^{2} x_{4} x_{5}^{2}, x_{1} x_{2} x_{3} x_{4} x_{5} x_{6}\right\rangle$.

\section{Connection to the Toric Hilbert Scheme}

In this section we explain the relevance of flips for the toric Hilbert scheme $H_{A}$. We begin by describing the toric Hilbert scheme.

A parameter space for the set of $A$-graded ideals was first described by Sturmfels [21]. Peeva and Stillman improved on this construction by producing the toric Hilbert scheme of $A$ [14], [15], which they show satisfies an important universal property. It is a version of their equations we explain below.

A degree $b \in \mathbb{N} A$ is a Graver degree if there is some Graver binomial $x^{\alpha}-x^{\beta}$ with $A \alpha=A \beta=b$. We denote by $b_{1}, \ldots, b_{N}$ the Graver degrees and by $m_{i}$ the number of monomials of degree $b_{i}$. Let

$$
X=\mathbb{P}^{m_{1}-1} \times \mathbb{P}^{m_{2}-1} \times \cdots \times \mathbb{P}^{m_{N}-1} .
$$

We now describe $H_{A}$ as a subscheme of $X$. The coordinates of each $\mathbb{P}^{m_{i}-1}$ can be labeled by the monomials of degree $b_{i}$ as $\left\{\xi_{u}: A u=b_{i}\right\}$. A point $p \in X$ corresponds to a weakly $A$-graded ideal $I_{p}$ by the following procedure: For each pair $x^{u}, x^{v}$ of degree $b_{i}$, we place the binomial $\xi_{v} x^{u}-\xi_{u} x^{v}$ in $I_{p}$. For each Graver binomial $x^{\alpha}-x^{\beta}$ there thus is either a binomial of the form $x^{\alpha}-c x^{\beta}$ in the resulting ideal, where $c$ may be zero, or $x^{\beta}=x^{\beta}-0 x^{\alpha}$ is in the ideal. This is immediate except in the case that 
$\xi_{\alpha}=\xi_{\beta}=0$. In that case, choose $\gamma$ with $A \gamma=A \alpha$ such that $\xi_{\gamma} \neq 0$. Then the binomial $\xi_{\gamma} x^{\alpha}-\xi_{\alpha} x^{\gamma} \in I_{p}$, so $x^{\alpha} \in I_{p}$, and $x^{\alpha}-0 \cdot x^{\beta}$ is the required binomial. Lemma 2.6 now implies that $I_{p}$ is weakly $A$-graded.

We note that the toric ideal $I_{A}$ corresponds to the point in $X$ with $\xi_{u}=1$ for all $u$. A monomial $A$-graded ideal corresponds to a point $p$ in $X$ where, for each $1 \leq i \leq N, p$ restricted to the $\mathbb{P}^{m_{i}-1}$ factor has one coordinate $\xi_{u_{i}}=1$, and all other $\xi_{v}$ in this factor equal to zero. In general, if $A u=b_{i}$, then $x^{u} \in I_{p}$ exactly if $\xi_{u}=0$.

We now give equations for $H_{A}$, which guarantee that the resulting ideals $I_{p}$ are in fact $A$-graded. For each $b \in \mathbb{N} A$ we construct the matrix $M_{b}$ whose $d_{b}$ rows are labeled by the monomials of degree $b$. The $n_{b}$ columns of $M_{b}$ are labeled by pairs $x^{u}, x^{v}$ of degree $b$ such that there is some binomial $x^{\alpha}-x^{\beta}$ whose degree is a Graver degree such that $u=v-\alpha+\beta$. The corresponding column consists of $\xi_{\alpha}$ in the $x^{u}$ row, $-\xi_{\beta}$ in the $x^{v}$ row, and zeros elsewhere.

The global equations for $H_{A}$ are now given by the maximal minors of $M_{b}$ for every $b \in \mathbb{N} A$. To see that these equations guarantee that $I_{p}$ is $A$-graded, note that if $I_{p}$ is not $A$-graded, there is some degree $b \in \mathbb{N} A$ with all monomials of degree $b$ contained in $I_{p}$. Now homogeneous polynomials of degree $b$ are in one-to-one correspondence with vectors in $k^{d_{b}}$. The bijection takes the basis vector with a one in the row corresponding to $x^{u}$ and zeros elsewhere to $x^{u}$, and is defined on other vectors by linear extension. Homogeneous polynomials of degree $b$ contained in $I_{p}$ are those corresponding to the image of the map $\sigma: k^{n_{b}} \rightarrow k^{d_{b}}$ given by $\sigma: x \mapsto M_{b} x$. Thus if all monomials of degree $b$ are in $I_{p}, M_{b}$ must have full rank, which means that there is a maximal minor which does not vanish.

While these equations for $H_{A}$ are not binomial, it follows from [14] that each irreducible component of the scheme is given by binomial equations. In [14] Peeva and Stillman give an explicit binomial description of the local equations for $H_{A}$ around each monomial ideal. The work of Eisenbud and Sturmfels on binomial ideals [5] now implies that the radical of the ideal defining each component is also a binomial ideal, and so the reduced structure on each irreducible component is a toric variety. It should be emphasized here that the components are not necessarily normal, and we are using a wider definition of toric variety than that found, for example, in [7]. We denote by $\tilde{H}_{A}$ the underlying reduced scheme of $H_{A}$.

The main result of this section is:

Theorem 3.1. The toric Hilbert scheme $H_{A}$ is connected if and only if the flip graph of $A$ is connected.

The remainder of this section builds up to the proof of Theorem 3.1. By the support of a point $v \in \mathbb{A}^{n}$ we mean $\operatorname{supp}(v):=\left\{i: v_{i} \neq 0\right\}$. In what follows we assume some familiarity with toric varieties, such as that given in [6] or [7].

Corollary 2.6 of [5] says that every prime binomial ideal determines a (not necessarily normal) toric variety. The next lemma gives a property of such varieties. When $Q$ is a prime ideal of $S$ we denote by $V(Q)$ the zero set of $Q$ in $\mathbb{A}^{n}$.

Lemma 3.2. Consider the point configuration $\left\{p_{1}, \ldots, p_{n}\right\} \subseteq \mathbb{Z}^{d}$ and its toric ideal $Q=\operatorname{ker}\left(\varphi: k\left[x_{1}, \ldots, x_{n}\right] \rightarrow k\left[t^{p_{1}}, \ldots, t^{p_{n}}\right]\right)$ which is a prime binomial ideal. Let $v_{1}$ 
and $v_{2}$ be two points in $V(Q) \subseteq \mathbb{A}^{n}$. Then $v_{1}$ and $v_{2}$ lie in the same torus orbit of $V(Q)$ if and only if they have the same support.

Proof. The dense torus in $V(Q)$ is $V(Q) \cap\left(k^{*}\right)^{n}$, and the action of this torus on $V(Q)$ is by coordinatewise multiplication. It thus follows that if $v_{1}$ and $v_{2}$ are in the same torus orbit, they have the same support.

Suppose $v_{1}, v_{2} \in V(Q)$ have the same support. If this support is the entire set $\{1, \ldots, n\}$, then define $u_{i}=\left(v_{1}\right)_{i} /\left(v_{2}\right)_{i}$. Then if $x^{\alpha}-x^{\beta}$ is a binomial in $Q, u^{\alpha}-u^{\beta}=$ $v_{1}^{\alpha} / v_{2}^{\alpha}-v_{1}^{\beta} / v_{2}^{\beta}=\left(1 / v_{2}^{\alpha} v_{2}^{\beta}\right)\left(v_{1}^{\alpha} v_{2}^{\beta}-v_{1}^{\beta} v_{2}^{\alpha}\right)=0$, so $u$ is in $V(Q) \cap\left(k^{*}\right)^{n}$, and so $v_{1}$ and $v_{2}$ are in the same torus orbit.

Suppose now that $v_{1}$ and $v_{2}$ have the same support $\tau \subsetneq\{1, \ldots, n\}$. Since $v_{1}$ and $v_{2}$ are in $V(Q)$, this means that there is no binomial in $Q$ of the form $x^{\alpha}-x^{\beta}$ where $\operatorname{supp}(\alpha) \subseteq \tau$ and $\operatorname{supp}(\beta) \nsubseteq \tau$. This is because if such a binomial were in $Q$, we will have $v_{i}^{\beta}=0$ for $i=1,2$, and $v_{i}^{\alpha} \neq 0$ for $i=1,2$, which contradicts $v_{1}, v_{2} \in V(Q)$. This means that there is no affine dependency between $\left\{p_{i}: i \in \tau\right\}$ and $\left\{p_{i}: i \notin \tau\right\}$. However, this implies that $\operatorname{conv}\left(p_{i}: i \in \tau\right)$ is a face of $\operatorname{conv}\left(p_{i}: 1 \leq i \leq n\right)$, and if $p_{j} \in \operatorname{conv}\left(p_{i}: i \in \tau\right)$, then $j \in \tau$. This means that $v_{1}$ and $v_{2}$ lie in an invariant toric subvariety, and so by a similar argument to above are torus isomorphic.

The action of $\left(k^{*}\right)^{n}$ on $A$-graded ideals gives an action of $\left(k^{*}\right)^{n}$ on $\tilde{H}_{A}$. The $n$-torus acts by mapping $v \in \tilde{H}_{A}$ to $t \cdot v$ via the map $(t \cdot v)_{u}=t^{u} v_{u}$. We refer to this action as the $n$-torus action. Since each irreducible component $V$ of $\tilde{H}_{A}$ is a toric variety, there is also an action of a $\operatorname{dim}(V)$-dimensional torus on a point for every irreducible component $V$ of the reduced toric Hilbert scheme the point belongs to. We refer to these actions as the ambient torus actions. We note that these torus actions are usually different from the $n$-torus action, as each of the finitely many irreducible components of $H_{A}$ has only finitely many ambient torus orbits, but there can be an infinite number of $n$-torus orbits. An example of this situation is given in Theorem 10.4 of [22]. The $n$-torus orbit of a point is, however, contained inside all ambient torus orbits of that point.

Corollary 3.3. Let $v$ be a point on $\tilde{H}_{A}$. Then the $n$-torus orbit of $v$ is contained in any ambient torus orbit of $v$.

Proof. It is straightforward to see that $t \cdot v$ lies in every irreducible component of $\tilde{H}_{A}$ in which $v$ does (this follows from the fact that $S[l] /\left(l I_{v}+(1-l)\left(I_{t \cdot v}\right)\right)$ is a flat $k[l]$ module). All points in the $n$-torus orbit of $v$ have the same support, and thus lie on the same ambient torus orbit by Lemma 3.2.

Fix an irreducible component $V$ of $\tilde{H}_{A}$. Since $V$ is a projective toric variety, there is a polytope $P$ corresponding to $V$. An ambient torus orbit of a point $v \in \tilde{H}_{A}$ corresponds to a face of $P$. In the case of the coherent component, this polytope is the state polytope of $I_{A}$. As the coherent component is not necessarily normal [20], however, the toric variety traditionally associated to the state polytope is only the normalization of the coherent component. The next two lemmas build up to showing that the edges of the polytope $P$ correspond exactly to flips. 
Lemma 3.4. Vertices of $P$ correspond exactly to the monomial A-graded ideals in $V$.

Proof. Let $I$ be the ideal corresponding to a vertex $p$ of $P$. The orbit of $I$ under the ambient torus corresponding to $P$ is just the ideal $I$. By Corollary 3.3 the $n$-torus orbit of $I$ is contained in any ambient torus orbit, so $I$ is $n$-torus fixed as well, and thus is a monomial ideal.

For the other implication, let $I$ be a monomial $A$-graded ideal corresponding to a point $v$ in $V$. As a point in $X, v$ is the product of unit vectors. It is thus invariant under any scaling of its coordinates in any fashion, and so is invariant under any ambient torus action. The point $v$ is therefore a vertex of $P$.

Lemma 3.5. Let I be an A-graded ideal. If I has exactly two initial ideals, then I is $n$-torus isomorphic to an ideal of the form $J=\left\langle x^{\alpha}-x^{\beta}, x^{\gamma_{1}}, \ldots, x^{\gamma_{r}}\right\rangle$.

Proof. Let $M_{1}$ and $M_{2}$ be the two initial ideals of $I$, and let $\mathcal{G}$ be the universal Gröbner basis of $I$. The set $\mathcal{G}$ contains a reduced Gröbner basis for $I$ with respect to a term order for which $M_{1}$ is the initial ideal, and so there exist binomials $x^{\alpha}-c x^{\beta} \in \mathcal{G}$ with $c \neq 0$ for which $x^{\alpha}$ is a minimal generator of $M_{1}, x^{\beta} \notin M_{1}$. Suppose for all such binomials we have $x^{\alpha} \in M_{2}$. Then $M_{1} \subseteq M_{2}$ is an inclusion of distinct monomial $A$-graded ideals, which is impossible. So we conclude that there is some binomial $x^{\alpha_{1}}-c_{1} x^{\beta_{1}} \in \mathcal{G}$ with $c_{1} \neq 0, x^{\alpha_{1}} \in M_{1} \backslash M_{2}$, and $x^{\beta_{1}} \in M_{2} \backslash M_{1}$.

Suppose there is some other binomial $x^{\alpha_{2}}-c_{2} x^{\beta_{2}} \in \mathcal{G}$ with $c_{2} \neq 0$. Without loss of generality we may assume that $x^{\alpha_{2}} \in M_{1}$ and $x^{\beta_{2}} \notin M_{1}$. We note that $\left(\alpha_{1}-\beta_{1}\right) \neq$ $\left(\alpha_{2}-\beta_{2}\right)$, as by Lemma 2.6 the two binomials $x^{\alpha_{1}}-x^{\beta_{1}}$ and $x^{\alpha_{2}}-x^{\beta_{2}}$ are Graver binomials, and they must be distinct since $\mathcal{G}$ is the universal Gröbner basis of $I$. We can thus find a supporting hyperplane for $\operatorname{pos}\left(\alpha_{1}-\beta_{1}, \beta_{2}-\alpha_{2}\right)$, which intersects the cone only at the origin. This implies the existence of a vector $w$ which satisfies $w \cdot\left(\alpha_{1}-\beta_{1}\right)>0$ and $w \cdot\left(\beta_{2}-\alpha_{2}\right)>0$. Let $M=\operatorname{in}_{w}(I)$. This is well defined as the Gröbner region of $I$ is all of $\mathbb{R}^{n}$. Then $x^{\alpha_{1}} \in M$ and $x^{\beta_{2}} \in M$, so $M \neq M_{1}$ and $M \neq M_{2}$. This means that $I$ has a third initial ideal, which contradicts our assumption, and so we conclude that $x^{\alpha_{1}}-c_{1} x^{\beta_{1}}$ is the only binomial in $\mathcal{G}$.

Pick $i \in \operatorname{supp}\left(\beta_{1}\right)$. Define $\lambda_{i}=1 / c_{1}$, and $\lambda_{j}=1$ for $j \neq i$. Then $\lambda I$ is in the desired form.

Theorem 3.6. Let $M_{1}$ and $M_{2}$ be monomial A-graded ideals corresponding to vertices $p_{1}$ and $p_{2}$ of $P . M_{1}$ and $M_{2}$ are connected by a single flip if and only if there is an edge e of $P$ connecting $p_{1}$ and $p_{2}$.

Proof. Suppose $p_{1}$ and $p_{2}$ are connected by an edge $e$. Let $I$ be the ideal corresponding to a point $p$ in the relative interior of $e$. By Corollary 3.3 the $n$-torus closure of $p$ is contained in $e$. Thus $I$ has at most two initial ideals. If $I$ had only one initial ideal, it would be a monomial ideal and thus corresponds to a vertex of $P$, by Lemma 3.4. We thus conclude that $I$ has exactly two initial ideals, $M_{1}$ and $M_{2}$, corresponding to $p_{1}$ and $p_{2}$, respectively. Now by Lemma $3.5 I$ is $n$-torus isomorphic to $J=\left\langle x^{\alpha}-x^{\beta}, x^{\gamma_{1}}, \ldots, x^{\gamma_{r}}\right\rangle$, where $x^{\alpha} \in M_{1} \backslash M_{2}$ and $x^{\beta} \in M_{2} \backslash M_{1}$. Since $J$ is $A$-graded, $x^{\alpha}-x^{\beta}$ is a Graver binomial. 
Because $J$ has initial ideals $M_{1}$ and $M_{2}$, it is their wall ideal $W_{\alpha-\beta}$, and so $M_{1}$ and $M_{2}$ are connected by a flip over $x^{\alpha}-x^{\beta}$.

Conversely, suppose $M_{1}$ and $M_{2}$ are connected by a single flip. Then there is an ideal $W_{\alpha-\beta}=\left\langle x^{\alpha}-x^{\beta}, x^{\gamma_{1}}, \ldots, x^{\gamma_{r}}\right\rangle$ which has as its two initial ideals $M_{1}$ and $M_{2}$. Let $J$ be an $A$-graded ideal which is isomorphic to $W_{\alpha-\beta}$ under the action of the ambient torus corresponding to $P$. Let $x^{\delta}$ be a minimal generator of $M_{1}$, with $\delta \neq \alpha$, and $x^{\delta}-x^{\varepsilon}$ the corresponding Graver binomial with $x^{\varepsilon} \notin M_{1}$. Then $x^{\delta} \in W_{\alpha-\beta}$, and thus $x^{\delta} \in J$, as the ambient torus action preserves the monomials in an $A$-graded ideal. So $J$ contains all minimal generators of $M_{1}$ and $M_{2}$ except $x^{\alpha}$ and $x^{\beta}$. Suppose $J$ has a minimal generator $x^{\alpha^{\prime}}-c x^{\beta^{\prime}}$, where $x^{\alpha^{\prime}}-x^{\beta^{\prime}}$ is a Graver binomial, $x^{\alpha^{\prime}}, x^{\beta^{\prime}} \notin J$, and $\alpha^{\prime}, \beta^{\prime} \neq \alpha, \beta$. Without loss of generality we may assume that $x^{\alpha^{\prime}} \in M_{1}$. If $x^{\beta^{\prime}} \notin M_{1}$, then $x^{\alpha^{\prime}} \in W_{\alpha-\beta}$ by the definition of $W_{\alpha-\beta}$, and thus also $x^{\alpha^{\prime}} \in J$. We thus conclude that $x^{\beta^{\prime}} \in M_{1}$. However, this means there exist $\alpha^{\prime \prime} \leq \alpha^{\prime}, \beta^{\prime \prime} \leq \beta^{\prime}$, such that $x^{\alpha^{\prime \prime}}$ and $x^{\beta^{\prime \prime}}$ are minimal generators of $M_{1}$. Since $x^{\alpha^{\prime}}$ and $x^{\beta^{\prime}}$ have disjoint support, we cannot have $\alpha^{\prime \prime}=\beta^{\prime \prime}=\alpha$, so at least one of $x^{\alpha^{\prime \prime}}$ and $x^{\beta^{\prime \prime}}$ is in $W_{\alpha-\beta}$. However, this means at least one of $x^{\alpha^{\prime}}$ and $x^{\beta^{\prime}}$ is in $J$, giving a contradiction. Hence the only binomial minimal generator of $J$ is of the form $x^{\alpha}-c^{\prime \prime} x^{\beta}$, so as in the proof of Lemma $3.5 J$ is $n$-torus isomorphic to $W_{\alpha-\beta}$. We thus see that all ambient torus closures of $W_{\alpha-\beta}$ are the same as the $n$-torus closure, and so $p_{1}$ and $p_{2}$ are connected by an edge.

Proof of Theorem 3.1. It suffices to show that the reduced scheme $\tilde{H}_{A}$ is connected if and only if the flip graph of $A$ is connected. Since passing to an initial ideal is a flat deformation, each irreducible component contains a monomial $A$-graded ideal. It thus suffices to show that all monomial $A$-graded ideals lie in the same connected component of $\tilde{H}_{A}$ if and only if the flip graph is connected. The "if" direction follows from the fact that if $I_{1}$ and $I_{2}$ are connected by a single flip, then they are both initial ideals of a single wall ideal $W_{\alpha-\beta}$, and so lie in the same connected component of $\tilde{H}_{A}$. The "only-if" direction follows from Lemma 3.4 and Theorem 3.6, which imply that the flip graph restricted to an irreducible component of $\tilde{H}_{A}$ is the edge skeleton of the polytope corresponding to that component, whose vertices are the monomial $A$-graded ideals in that component, and so is connected. As the intersection of two irreducible components

of $\tilde{H}_{A}$ contains a monomial $A$-graded ideal by Gröbner deformation, this means that if $\tilde{H}_{A}$ is connected, the flip graph of $A$ is connected.

Remark 3.7. We close this section by noting that Peeva and Stillman [15] have shown that the dimension of the tangent space to $H_{A}$ at a monomial $A$-graded ideal $I$ is equal to the number of flippable binomials of $I$.

\section{Toric Hilbert Schemes of Arbitrarily High Dimension from Curves in $\mathbb{P}^{4}$}

In this section we exhibit toric Hilbert schemes of arbitrarily high dimensions for which the associated toric varieties are curves in $\mathbb{P}^{4}$. When $A$ has corank one, its Graver basis consists of precisely one binomial $x^{\alpha}-x^{\beta}$, and the flip graph of $A$ has only the two vertices $I=\left\langle x^{\alpha}\right\rangle$ and $I^{\prime}=\left\langle x^{\beta}\right\rangle$ which are connected by the flip $x^{\alpha}-x^{\beta}$. Hence $H_{A}$ is one- 
dimensional and connected. All $A$-graded ideals of a corank two matrix are coherent [8] which implies that the flip graph of $A$ is connected since it coincides with the edge graph of the state polytope of $I_{A}$. In this case, $H_{A}$ has exactly one irreducible component which is two-dimensional and smooth [15]. The toric Hilbert scheme of a corank three matrix is at least three-dimensional since the irreducible component containing the coherent $A$-graded ideals has dimension three. In contrast to the results in coranks one and two, Theorem 4.1 gives a family of $2 \times 5$ matrices of corank three whose toric Hilbert schemes can have arbitrarily high dimensions. The projective toric variety of each matrix in the family is a curve in $\mathbb{P}^{4}$. Note that both the corank $n-d$ and the number of columns $n$ are fixed for these matrices.

Theorem 4.1. For each $j \in \mathbb{N} \backslash\{0\}$, the toric Hilbert scheme $H_{A(j)}$ of

$$
A(j)=\left(\begin{array}{ccccc}
1 & 1 & 1 & 1 & 1 \\
0 & 1 & 3+3 j & 4+3 j & 6+3 j
\end{array}\right)
$$

has an irreducible component of dimension at least $j$.

These matrices were motivated by Example 5.11 in [23], and the theorem was inspired by computer experiments on their flip graphs. We first define the following monomial ideals and sets of binomials that will be used in the proof of Theorem 4.1. For each $j \in \mathbb{N} \backslash\{0\}$, let

$$
\begin{aligned}
P_{j} & =\left\langle c^{2} e, b c, a^{2} e, a c e, a e^{j+2}\right\rangle, & R_{j} & =\left\langle a^{5} c^{j}, a^{8} c^{j-1}, \ldots, a^{5+3(j-1)} c\right\rangle, \\
Q_{j} & =\left\langle b e^{j+1}, a^{2} c^{j+1}, b^{4} e^{j}, c^{j+2}\right\rangle, & S_{j} & =\left\langle b^{7} e^{j-1}, b^{10} e^{j-2}, \ldots, b^{7+3(j-1)}\right\rangle
\end{aligned}
$$

and

$$
\begin{aligned}
\mathcal{P}_{j} & =\left\{c^{2} e-d^{3}, b c-a d, a^{2} e-b^{2} d, a c e-b d^{2}, a e^{j+2}-c^{j} d^{3}\right\}, \\
\mathcal{Q}_{j} & =\left\{b e^{j+1}-c^{j+1} d, a^{2} c^{j+1}-b^{3} e^{j}, b^{4} e^{j}-a^{3} c^{j} d, c^{j+2}-a e^{j+1}\right\}, \\
\mathcal{R}_{j} & =\left\{a^{5+3 t} c^{j-t}-b^{6+3 t} e^{(j-1)-t}, t=0,1, \ldots, j-1\right\}, \\
\mathcal{S}_{j} & =\left\{b^{7+3 t} e^{(j-1)-t}-a^{6+3 t} c^{(j-1)-t} d, t=0,1, \ldots, j-1\right\} .
\end{aligned}
$$

Lemma 4.2. The ideal $M_{j}=P_{j}+Q_{j}+R_{j}+S_{j}$ is the initial ideal of $I_{A(j)}$ with respect to the weight vector $w=(1,1,2,0,2)$.

Proof. By computing the $A(j)$-degree of both terms in each binomial of $\mathcal{G}_{j}:=\mathcal{P}_{j} \cup$ $\mathcal{Q}_{j} \cup \mathcal{R}_{j} \cup \mathcal{S}_{j}$, it can be seen that $\mathcal{G}_{j}$ is a subset of $I_{A(j)}$. It can also be checked that for each binomial in $\mathcal{G}_{j}$, the positive term is the leading term with respect to $w=(1,1,2,0,2)$. Hence $M_{j}=\left\langle\operatorname{in}_{w}(g): g \in \mathcal{G}_{j}\right\rangle$ is contained in the initial ideal of $I_{A(j)}$ with respect to $w$ and no generator of $M_{j}$ is redundant. The monomial ideal $M_{j}$ will equal $\operatorname{in}_{w}\left(I_{A(j)}\right)$ if $\mathcal{G}_{j}$ is the reduced Gröbner basis of $I_{A(j)}$ with respect to $w$. Consider the elimination order $x, y \succ a, b, c, d, e$ refined by the graded reverse lexicographic order $x>y$ on the first block of variables and the weight vector $w$ on the second block of variables. Then 
the reduced Gröbner basis of $I_{A(j)}$ with respect to $w$ is the intersection of the reduced Gröbner basis of

$$
J(j):=\left\langle a-x, b-x y, c-x y^{3+3 j}, d-\subset x y^{4+3 j}, e-x y^{6+3 j}\right\rangle
$$

with respect to $\succ$ with $k[a, b, c, d, e]$ (see Algorithm 4.5 in [22]). By a laborious check it can be shown that the reduced Gröbner basis of $J(j)$ with respect to $\succ$ is

$$
\begin{aligned}
& \mathcal{G}_{j} \cup\left\{x-a, y a-b, y b d-a e, y c-d, y d^{2}-c e, y^{2} d-e,\right. \\
& y b^{3 t+2} e^{j-t}-a^{3 t+1} c^{(j+1)-t}, t=0, \ldots, j, \\
& y^{2} b^{3 t+1} e^{j-t}-a^{3 t} c^{(j+1)-t}, t=0, \ldots, j, \\
& y^{3 l} b^{3 t} e^{p_{l}-t}-a^{3 t-1} c^{p_{l}-t+1}, l=1, \ldots, j, t=1, \ldots, p_{l}:=(j+1)-l, \\
& y^{3 l+1} b^{3 t-1} e^{p_{l}-t}-a^{3 t-2} c^{p_{l}-t+1}, l=1, \ldots, j, t=1, \ldots, p_{l}, \\
&\left.y^{3 l+2} b^{3 t-2} e^{p_{l}-t}-a^{3 t-3} c^{p_{l}-t+1}, l=1, \ldots, j, t=1, \ldots, p_{l}\right\} .
\end{aligned}
$$

Lemma 4.3. For each $j \in \mathbb{N} \backslash\{0\}$ the monomial $A(j)$-graded ideal $M_{j}$ from Lemma 4.2 has exactly $2 j+4$ flippable binomials.

Proof. We will show that the binomials in $\mathcal{Q}_{j} \cup \mathcal{R}_{j} \cup \mathcal{S}_{j}$ are flippable for $M_{j}$ while those in $\mathcal{P}_{j}$ are not. In order to show that a binomial $x^{\alpha}-x^{\beta}$ is flippable for $M_{j}$ we need to show that every $S$-polynomial (monomial in our case) formed from the binomial $x^{\alpha}-x^{\beta}$ (with $x^{\alpha}$ as leading term) and a minimal generator $x^{\gamma}$ of $M_{j}$ different from $x^{\alpha}$ reduces to zero modulo $W_{\alpha-\beta}=\left\langle x^{\gamma}: \gamma \neq \alpha, x^{\gamma}\right.$ a minimal generator of $\left.M_{j}\right\rangle+\left\langle x^{\alpha}-x^{\beta}\right\rangle$.

We first consider $\mathcal{R}_{j}$. A binomial $a^{5+3 t} c^{j-t}-b^{6+3 t} e^{(j-1)-t}$ in $\mathcal{R}_{j}$ can form a non-trivial $S$-pair ( $S$-monomial) with

(i) $c^{2} e$,

(ii) $b c$,

(iii) $a^{2} e$,

(iv) ace,

(v) $a e^{j+2}$,

(vi) $a^{2} c^{j+1}$,

(vii) $c^{j+2}$, and

(viii) a monomial $a^{5+3 l} c^{j-l}$ from $R_{j}$ such that $t \neq l$.

The remaining generators of $M_{j}$ (except $a^{5+3 t} c^{j-t}$ itself) are relatively prime to $a^{5+3 t} c^{j-t}$ and so the $S$-pairs formed reduce to zero by Buchberger's first criterion. We consider each case separately.

(i) The $S$-monomials formed from $c^{2} e$ and $a^{5+3 t} c^{j-t}-b^{6+3 t} e^{(j-1)-t}$ are $b^{6+3 t} c^{p} e^{j-t}$, $0 \leq t \leq j-1$, where $p=1$ if $j-t=1$ and $p=0$ if $j-t>1$.

(a) If $t=0, b^{6} c^{p} e^{j}$ is a multiple of $b^{4} e^{j} \in Q_{j}$.

(b) If $t>0, b^{6+3 t} c^{p} e^{j-t}$ reduces to zero modulo $b^{7+3(t-1)} e^{j-t} \in S_{j}$.

(ii) The $S$-monomials formed from $b c$ are $b^{7+3 t} e^{(j-1)-t}, 0 \leq t \leq j-1$, all of which lie in $S_{j}$ and hence reduce to zero modulo $W_{\alpha-\beta}$. 
(iii) The $S$-monomials between $a^{2} e$ and $a^{5+3 t} c^{j-t}-b^{6+3 t} e^{(j-1)-t}$ are $b^{6+3 t} e^{j-t}$ for $0 \leq t \leq j-1$. If $t=0$, then $b^{6} e^{j}$ is a multiple of $b^{4} e^{j} \in Q_{j}$, and if $t>0$, then $b^{6+3 t} e^{j-t}$ is divisible by $b^{7+3(t-1)} e^{j-t} \in S_{j}$.

(iv) The $S$-monomials from ace are $b^{6+3 t} e^{j-t}$ for $0 \leq t \leq j-1$, all of which reduce to zero as in (iii).

(v) The monomial $a e^{j+2}$ gives $b^{6+3 t} e^{2 j+1-t}$ for $0 \leq t \leq j-1$, all of which reduce to zero modulo $b e^{j+1} \in Q_{j}$.

(vi) From $a^{2} c^{j+1}$ we get $b^{6+3 t} c^{t+1} e^{(j-1)-t}, 0 \leq t \leq j-1$, all of which are multiples of $b c \in P_{j}$.

(vii) The $S$-monomials from $c^{j+2}$ are $b^{6+3 t} c^{t+2} e^{(j-1)-t}$ which are also multiples of $b c \in P_{j}$ for $0 \leq t \leq j-1$.

(viii) For this last case, suppose first that $l<t \in\{0,1,2, \ldots, j-1\}$. Then $\operatorname{lcm}\left(a^{5+3 l} c^{j-l}, a^{5+3 t} c^{j-t}\right)=a^{5+3 t} c^{j-l}$ and the $S$-monomial between $a^{5+3 l} c^{j-l}$ and $a^{5+3 t} c^{j-t}-b^{6+3 t} e^{(j-1)-t}$ is $b^{6+3 t} c^{t-l} e^{(j-1)-t}$ which is a multiple of $b c \in P_{j}$. If $l>t$, then the $S$-monomial is $a^{3(l-t)} b^{6+3 t} e^{(j-1)-t}$ which is divisible by $a^{2} e \in P_{j}$ since $t<l \leq j-1$ and hence $t<j-1$.

Similarly, one can check that the binomials in $\mathcal{Q}_{j} \cup \mathcal{S}_{j}$ are all flippable for $M_{j}$, which shows that $M_{j}$ has at least $2 j+4$ flippable binomials. To finish the proof, we argue that no binomial in $\mathcal{P}_{j}$ is flippable for $M_{j}$.

(i) The $S$-binomial between $c^{2} e-d^{3} \in \mathcal{P}_{j}$ and $b c \in P_{j}$ is $b d^{3}$ which is not divisible by any generator of $M_{j}$.

(ii) The binomials $b c-a d, a^{2} e-b^{2} d$, and $a c e-b d^{2} \in \mathcal{P}_{j}$ form the $S$-binomials $a d e^{j+1}, b^{3} d e^{j}$, and $b^{2} d^{2} e^{j}$, respectively with $b e^{j+1} \in Q_{j}$. None of them can be divided by a minimal generator of $M_{j}$.

(iii) The $S$-binomial of $a e^{j+2}-c^{j} d^{3} \in \mathcal{Q}_{j}$ and $a^{2} e \in P_{j}$ is $a c^{j} d^{3}$ which does not lie in $M_{j}$.

Hence $M_{j}$ has exactly $2 j+4$ flippable binomials.

Proof of Theorem 4.1. The same proof as in Lemma 4.3 shows that the generators of $\mathcal{I}\left(\mu_{0}, \ldots, \mu_{j-1}\right):=P_{j}+Q_{j}+\left\langle a^{5+3 t} c^{j-t}-\mu_{t} b^{6+3 t} e^{j-1-t}, t=0, \ldots, j-1\right\rangle+S_{j}$ form a Gröbner basis with respect to $w=(1,1,2,0,2)$ with initial ideal $M_{j}$, for every choice of scalars $\mu_{0}, \ldots, \mu_{j-1}$ from the underlying field $k$. Lemma 4.3 proved this claim for the case when $\mu_{i}=1$ for some $0 \leq i \leq j-1$ and $\mu_{j}=0$ for all $j \neq i$. Since $M_{j}$ is $A(j)$-graded, the $A(j)$-homogeneous ideal $\mathcal{I}\left(\mu_{0}, \ldots, \mu_{j-1}\right)$ is also $A(j)$-graded for every choice of scalars $\mu_{0}, \ldots, \mu_{j-1}$. Hence there is an injective polynomial map from $\mathbb{A}_{k}^{j} \rightarrow H_{A(j)}$, such that $\left(\mu_{0}, \ldots, \mu_{j-1}\right)$ maps to the point on $H_{A(j)}$ corresponding (uniquely) to $\mathcal{I}\left(\mu_{0}, \ldots, \mu_{j-1}\right)$. Since $\mathbb{A}_{k}^{j}$ is irreducible, the image of this map lies entirely in one irreducible component of the toric Hilbert scheme $H_{A(j)}$ and the dimension of this component is at least $\operatorname{dim}\left(\mathbb{A}_{k}^{j}\right)=j$.

Remark 4.4. In [23] it was conjectured that the maximum valency of a vertex in the state polytope of $I_{A}$ is bounded above by a function of just the corank of $A$. As a particular case, it was also conjectured that if $A$ is of corank three, then every vertex in the state polytope of $I_{A}$ has at most four neighbors. This latter conjecture was recently 
disproved by Hoşten and Maclagan [10] who have found vertices with up to six neighbors. Lemma 4.3 shows that even in corank three, a vertex in the flip graph of $A$ can have arbitrarily many neighbors.

\section{Connection to the Baues Problem}

In this section we elaborate a connection between $A$-graded ideals and the Baues problem for triangulations. A good reference for all forms of the Baues problem is [17].

A triangulation of a point configuration $\mathcal{A}=\left\{a_{1}, \ldots, a_{n}\right\} \subseteq \mathbb{Z}^{d}$ is a geometric simplicial complex covering conv $\left(a_{1}, \ldots, a_{n}\right)$ with the vertices of each simplex being a subset of $\mathcal{A}$. Each simplex $\sigma$ is indexed by the set $\left\{i: a_{i}\right.$ is a vertex of $\left.\sigma\right\}$.

A basic operation on triangulations of a point configuration is the bistellar flip. The two basic types of non-degenerate bistellar flips in the plane are shown in Fig. 1.

Intuitively, a bistellar flip should be thought of as gluing in a higher-dimensional simplex, and then turning that simplex over and viewing it from the other side. This can be seen most clearly in the second example in Fig.1, which can be viewed as the top and bottom of a tetrahedron. The first example can also be thought of as two opposite views of a tetrahedron.

More formally, a bistellar flip interchanges the two different triangulations of a circuit of $\mathcal{A}$. A vector $t \in \mathbb{Z}^{n}$ is called a circuit of $\mathcal{A}$ if it lies in the kernel of the matrix whose columns are the points of $\mathcal{A}, \operatorname{supp}(t)$ is minimal with respect to inclusion when compared against the supports of all integral vectors in the kernel of this matrix, and its non-zero entries do not have a common divisor. Let $t$ be a circuit of the configuration $\mathcal{A}$, and let $T=\left\{i: t_{i} \neq 0\right\}$ be its support. We denote by $T^{+}$the set $\left\{i: t_{i}>0\right\}$ and by $T^{-}$the set $\left\{i: t_{i}<0\right\}$. There are exactly two triangulations of $C=\operatorname{conv}\left(a_{i}: i \in T\right)$. The first, $C^{+}$, has $\left|T^{+}\right|$simplices, which are the simplices indexed by the sets $\left\{T \backslash\{i\}: i \in T^{+}\right\}$. The second, $C^{-}$, has $\left|T^{-}\right|$simplices, which are the sets in $\left\{T \backslash\{i\}: i \in T^{-}\right\}$. The unique minimal non-face of $C^{+}\left(C^{-}\right)$is $T^{+}\left(T^{-}\right)$. If $C$ is $d$-dimensional, and one of $C^{+}$and $C^{-}$is a subcomplex of the triangulation $\Delta$, then a bistellar flip over the circuit $t$ involves replacing the subcomplex $C^{+}$by $C^{-}$or vice versa.

If $C$ is lower-dimensional, we impose an additional condition for $t$ to be flippable. By the link of a simplex $\sigma$ in a simplicial complex $\Delta$ we mean the collection of simplices $\{\tau: \tau \cap \sigma=\emptyset, \tau \cup \sigma \in \Delta\}$. We say $t$ is flippable if $C^{+}$(or $C^{-}$) is a subcomplex of

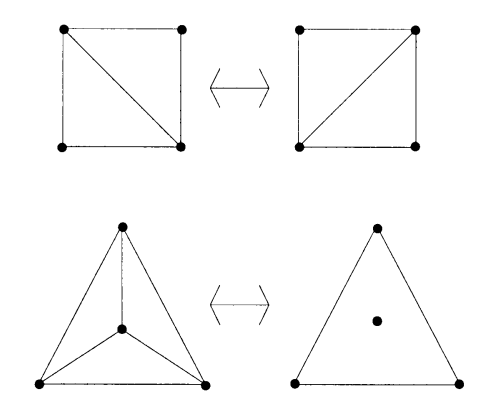

Fig. 1. Bistellar flips for triangulations of points in the plane. 


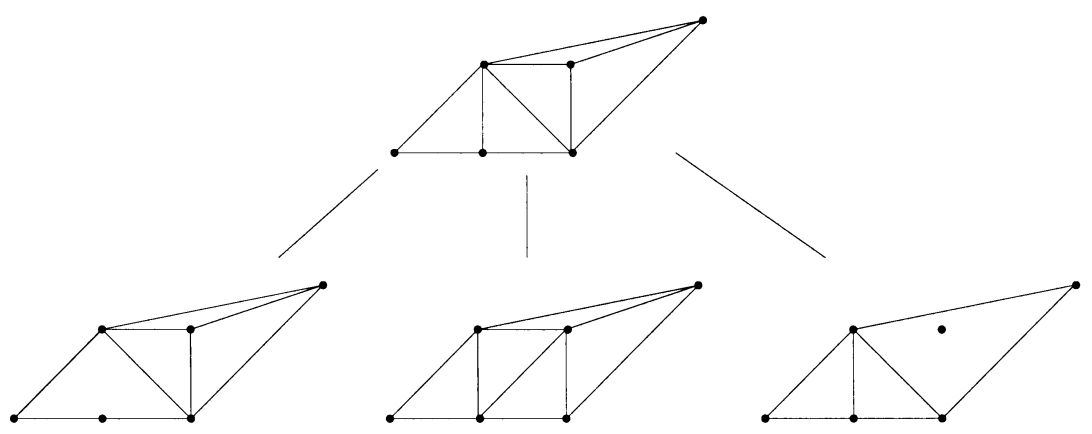

Fig. 2. Triangulations differing by bistellar flips.

$\Delta$, and the link in $\Delta$ of every maximal simplex of $C^{+}$(respectively $C^{-}$) is the same subcomplex $L$. This second condition is trivially satisfied if $C$ is $d$-dimensional, as the link of every maximal simplex is the empty set. A bistellar flip over the circuit $t$ from $C^{+}$to $C^{-}$then involves replacing the simplices $\left\{l \cup \sigma: \sigma \in C^{+}, l \in L\right\}$ by the simplices $\left\{l \cup \tau: \tau \in C^{-}, l \in L\right\}$.

Examples of bistellar flips are shown in Fig. 2.

We can form a graph, called the Baues graph, on the set of all triangulations of a point configuration, with an edge connecting two triangulations when they differ by a bistellar flip. Figure 2 is a subgraph of the Baues graph for a particular collection of six points in the plane. An obvious question to ask is whether the Baues graph is connected. Santos recently answered this question negatively [18], constructing a configuration of 324 points in $\mathbb{Z}^{6}$ which has a disconnected Baues graph. For the original Baues problem, the point configurations considered can have real coordinates and Santos's main point configuration lies in $\mathbb{R}^{6}$. However, he also exhibits a configuration in $\mathbb{Z}^{6}$ with 324 points that has a disconnected Baues graph.

The rest of this section relates the Baues graph to the flip graph and the toric Hilbert scheme. The connection is through the following lemma, which is a special case of Theorem 10.10 in [22]. It links monomial $A$-graded ideals and triangulations of $\mathcal{A}$, where $A$ is the matrix whose columns are the points in $\mathcal{A}$, with an additional row of ones added. We denote both the $i$ th column of $A$ and the $i$ th point of $\mathcal{A}$ by $a_{i}$. We adopt the notational convention that if $\sigma \subseteq\{1, \ldots, n\}$ is a set, then $x^{\sigma}=\prod_{i \in \sigma} x_{i}$. The Stanley-Reisner ideal (see [19]) $I(\Delta)$ of a simplicial complex $\Delta$ is the ideal generated by the monomials $x^{\sigma}$ where the sets $\sigma$ are the minimal non-faces of $\Delta$. Similarly, every squarefree monomial ideal $I$ in $S$ defines a unique simplicial complex $\Delta(I)$ on $\{1, \ldots, n\}$.

Lemma 5.1 [22, Theorem 10.10]. Let I be a monomial A-graded ideal. Then $\Delta(\mathrm{rad}$ $(I))$, the simplicial complex associated to $\operatorname{rad}(I)$ via the Stanley-Reisner correspondence, is a triangulation of $\mathcal{A}$.

We can now state the main theorem of this section.

Theorem 5.2. Let I be a monomial A-graded ideal and let $x^{\alpha}-x^{\beta}$ be a flippable binomial for $I$. Then either $\Delta(\operatorname{rad}(I))=\Delta\left(\operatorname{rad}\left(I_{\text {flip }}\right)\right)$ or they differ by a bistellar flip. 
The proof will be developed through the following series of lemmas. We need to show that if $I_{1}$ and $I_{2}$ are monomial $A$-graded ideals which differ by a flip, then either their radicals are the same or $\Delta\left(\operatorname{rad}\left(I_{1}\right)\right)$ and $\Delta\left(\operatorname{rad}\left(I_{2}\right)\right)$ differ by a bistellar flip. The latter involves showing:

(1) $t=\alpha-\beta$ is a circuit of $\mathcal{A}$ (Lemma 5.3).

(2) $C^{+}$is a subcomplex of $\Delta(\operatorname{rad}(I))$ with the link of all maximal simplices of $C^{+}$ the same (Lemma 5.4).

(3) $\Delta\left(\operatorname{rad}\left(I_{\text {flip }}\right)\right)$ differs from $\Delta(\operatorname{rad}(I))$ exactly by replacing $C^{+}$and its link by $C^{-}$ and the corresponding link.

By a circuit of $A$ we mean a binomial $x^{\alpha}-x^{\beta}$ such that $\alpha-\beta$ is a circuit of $\mathcal{A}$. The set of circuits is contained in the Graver basis of $A$ [22, Proposition 4.11].

Lemma 5.3. Let I be a monomial A-graded ideal, with $x^{\alpha}-x^{\beta}$ a fippable binomial with $x^{\alpha} \in I$. Then $x^{\beta} \in \operatorname{rad}(I) \Leftrightarrow \operatorname{rad}(I)=\operatorname{rad}\left(I_{\text {flip }}\right)$. If $x^{\beta} \notin \operatorname{rad}(I)$, then $x^{\alpha}-x^{\beta}$ is a circuit of $A$.

Proof. The implication $\Leftarrow$ is immediate in the first statement so we need only show that $x^{\beta} \in \operatorname{rad}(I)$ implies $\operatorname{rad}(I)=\operatorname{rad}\left(I_{\text {flip }}\right)$. Suppose $x^{\beta} \in \operatorname{rad}(I)$. Let $x^{\gamma}$ be a minimal generator of $I_{\text {flip }}$. Then either $x^{\gamma}$ is a minimal generator of $I, \gamma=\beta$, or $\gamma=\alpha+g$ for some $g$. In each case $x^{\gamma} \in \operatorname{rad}(I), \operatorname{so} \operatorname{rad}\left(I_{\text {flip }}\right) \subseteq \operatorname{rad}(I)$. If the containment is proper, Lemma 5.1 gives a proper containment of Stanley-Reisner ideals of triangulations of $\mathcal{A}$, which is not possible. So we conclude $\operatorname{rad}(I)=\operatorname{rad}\left(I_{\text {flip }}\right)$.

For the second statement, suppose $x^{\alpha}-x^{\beta}$ is not a circuit. Then there exists a circuit $x^{\gamma}-x^{\delta}$ with $\operatorname{supp}(\gamma) \subseteq \operatorname{supp}(\alpha)$, and $\operatorname{supp}(\delta) \subseteq \operatorname{supp}(\beta)$ where at least one of these inclusions is proper. Since $x^{\beta} \notin \operatorname{rad}(I)$, we must have $x^{\text {supp }(\delta)} \notin \operatorname{rad}(I)$, and thus $x^{\delta} \notin I$. This implies $x^{\gamma} \in I$. Because $x^{\alpha}-x^{\beta}$ is not a circuit, while $x^{\gamma}-x^{\delta}$ is, we know $\gamma \neq \alpha$, and thus $x^{\gamma} \in I_{\text {flip }}$. This means $x^{\text {supp }(\gamma)} \in \operatorname{rad}\left(I_{\text {flip }}\right)$, and so $x^{\alpha} \in \operatorname{rad}\left(I_{\text {flip }}\right)$. However, this means, as above, that $\operatorname{rad}\left(I_{\text {flip }}\right)=\operatorname{rad}(I)$, which in turn implies that $x^{\beta} \in \operatorname{rad}(I)$, contradicting the hypothesis.

Let $I$ be a monomial $A$-graded ideal, with $x^{\alpha}-x^{\beta}$ flippable, where $x^{\alpha} \in I, x^{\beta} \notin$ $\operatorname{rad}(I)$. Let $t=\alpha-\beta$ and $T=\operatorname{supp}(t)$. By Lemma 5.3 we know that $t$ is a circuit, so we can consider the triangulation $C^{+}=\left\{T \backslash\{i\}: i \in T^{+}\right\}$of $C$.

Lemma 5.4. Let $I, x^{\alpha}-x^{\beta}, t$, and $C^{+}$be as above. Then $C^{+}$is a subcomplex of $\Delta=\Delta(\operatorname{rad}(I))$, and there is a subcomplex of $\Delta$ which is the common link of all maximal simplices of $C^{+}$.

Proof. Since $T^{+}$is the only minimal non-face of $C^{+}$, to show that $C^{+}$is a subcomplex of $\Delta$ we need to show that $x^{T^{+}}$is the only minimal generator of $\operatorname{rad}(I)$ with support in $T$.

Suppose $x^{\gamma}$ is a minimal generator of $\operatorname{rad}(I)$, with $\operatorname{supp}(\gamma) \subseteq T$. Then there is some $l \geq 1$ such that $x^{l \gamma} \in I$. Write $\gamma=\alpha^{\prime}+\beta^{\prime}$, where $\operatorname{supp}\left(\alpha^{\prime}\right) \subseteq \operatorname{supp}(\alpha)$ and $\operatorname{supp}\left(\beta^{\prime}\right) \subseteq \operatorname{supp}(\beta)$. If $\operatorname{supp}\left(\alpha^{\prime}\right) \neq \operatorname{supp}(\alpha)$, then $x^{\alpha}$ does not divide $x^{l \gamma}$ and so $x^{l \gamma}$ is in 
the wall ideal $W_{\alpha-\beta}$. We can choose $\delta^{\prime}$ with $\operatorname{supp}\left(\delta^{\prime}\right) \subseteq \operatorname{supp}(\beta)$ so that $l \gamma+\delta^{\prime}=m \beta+\alpha^{\prime}$ for some $m \geq 1$. Since $x^{m \beta+\alpha^{\prime}}=x^{l \gamma+\delta^{\prime}} \in W_{\alpha-\beta}$, it follows that $x^{m \alpha+\alpha^{\prime}} \in W_{\alpha-\beta}$, because $x^{\alpha}-x^{\beta} \in W_{\alpha-\beta}$. So $x^{m \alpha+\alpha^{\prime}} \in I_{\text {flip }}$, and there is thus some $p \geq m+1$ such that $x^{p \alpha} \in I_{\text {flip }}$. This implies that $x^{\alpha} \in \operatorname{rad}\left(I_{\text {flip }}\right)$. However, by Lemma 5.3, this means that $\operatorname{rad}\left(I_{\text {flip }}\right)=\operatorname{rad}(I)$, which in turn implies that $x^{\beta} \in \operatorname{rad}(I)$, contradicting our hypothesis. So $\operatorname{supp}\left(\alpha^{\prime}\right)=\operatorname{supp}(\alpha)=T^{+}$, and thus $x^{T^{+}} \mid x^{\gamma}$. This shows that $x^{T^{+}}$is the only minimal generator of $\operatorname{rad}(I)$ with support in $T$, as required. From this we conclude that $C^{+}$is a subcomplex of $\Delta$.

We now show that every maximal simplex $\sigma \in C^{+} \subseteq \Delta$ has the same link. We do this by showing that any simplex not intersecting $T$ and not in the link of one maximal simplex of $C^{+}$is not in the link of any other maximal simplex of $C^{+}$.

Suppose $\sigma \subseteq\{1, \ldots, n\} \backslash T$ is not a simplex in the link of a maximal simplex $\gamma^{\prime}$ of $C^{+} \subseteq \Delta$, where $\gamma^{\prime}=T \backslash\{p\}$ for some $p \in T^{+}$and $\sigma \cap T=\emptyset$. Then $x^{\sigma \cup \gamma^{\prime}} \in \operatorname{rad}(I)$, because $\sigma \cup \gamma^{\prime}$ is not a face of $\Delta$, and so there exists $l \geq 1$, and $x^{\alpha^{\prime \prime}}$, a minimal generator of $I$ with $\alpha^{\prime \prime} \neq \alpha$, such that $x^{\alpha^{\prime \prime}} \mid\left(x^{\sigma \cup \gamma^{\prime}}\right)^{l}$. Write $\alpha^{\prime \prime}=\alpha^{\prime}+\beta^{\prime}+\sigma^{\prime}$, where $\operatorname{supp}\left(\alpha^{\prime}\right) \subseteq \operatorname{supp}(\alpha), \operatorname{supp}\left(\beta^{\prime}\right) \subseteq \operatorname{supp}(\beta)$, and $\operatorname{supp}\left(\sigma^{\prime}\right) \subseteq \sigma$. Choose $\delta^{\prime}$ with $\operatorname{supp}\left(\delta^{\prime}\right) \subseteq \operatorname{supp}(\alpha)$ such that $\alpha^{\prime \prime}+\delta^{\prime}=m \alpha+\beta^{\prime}+\sigma^{\prime}$ for some $m \geq 0$. Then because $x^{\alpha^{\prime \prime}} \in W_{\alpha-\beta}$, we have $x^{\alpha^{\prime \prime}+\delta^{\prime}} \in W_{\alpha-\beta}$, and so $x^{m \beta+\beta^{\prime}+\sigma^{\prime}}$ is in $W_{\alpha-\beta}$ and thus in $I$. So $x^{\operatorname{supp}(\beta) \cup \operatorname{supp}\left(\sigma^{\prime}\right)} \in \operatorname{rad}(I)$. Let $\tau$ be another maximal simplex of $C^{+}$, so $\tau=\left(\gamma^{\prime} \cup\{p\}\right) \backslash\left\{p^{\prime}\right\}$ for some $p^{\prime} \in T^{+}$. Then $\operatorname{supp}(\beta) \cup \operatorname{supp}\left(\sigma^{\prime}\right) \subseteq \tau \cup \sigma$, and so $x^{\tau \cup \sigma} \in \operatorname{rad}(I)$, and thus $\sigma$ is not in the link of $\tau$ in $\Delta$. This shows that every maximal simplex $\sigma \in C^{+} \subseteq \Delta$ has the same link, as required.

Proof of Theorem 5.2. If $x^{\beta} \in \operatorname{rad}(I)$, then $\operatorname{rad}(I)=\operatorname{rad}\left(I_{\text {flip }}\right)$ by Lemma 5.3, and so $\Delta(\operatorname{rad}(I))=\Delta\left(\operatorname{rad}\left(I_{\text {flip }}\right)\right)$.

Suppose $x^{\beta} \notin \operatorname{rad}(I)$. Then Lemma 5.3 implies that $t=\alpha-\beta$ is a circuit of $\mathcal{A}$. By Lemma $5.4 C^{+}$is a subcomplex of $\Delta(\operatorname{rad}(I))$ with each maximal simplex of $C^{+}$ having the same link in $\Delta(\operatorname{rad}(I))$. It remains to show that $\Delta\left(\operatorname{rad}\left(I_{\text {flip }}\right)\right)$ is the result of performing a bistellar flip on $\Delta(\operatorname{rad}(I))$.

Let $\Delta^{\prime}$ be the result of performing the bistellar flip on $\Delta(\operatorname{rad}(I))$ over $t$, and let $M$ be the Stanley-Reisner ideal of $\Delta^{\prime}$.

We claim that $M$ is the squarefree monomial ideal generated by $x^{\operatorname{supp}(\beta)}$, all the generators of $\operatorname{rad}(I)$ except $x^{\operatorname{supp}(\alpha)}$, and also all monomials of the form $x^{\sigma}$, such that $\operatorname{supp}(\alpha) \subseteq \sigma$, and $\sigma \backslash(T \cap \sigma)$ is not in the link of the maximal simplices of $C^{+}$. Let $\alpha^{\prime \prime} \subseteq\{1, \ldots, n\}$. Then $\alpha^{\prime \prime}$ is a face of $\Delta^{\prime}$ exactly when either $\alpha^{\prime \prime}$ is a face of $\Delta$ and $T^{-} \nsubseteq \alpha^{\prime \prime}$ or $\alpha^{\prime \prime}=T^{+} \cup \tau \cup \gamma^{\prime}$, where $\tau \subsetneq T^{-}$, and $\gamma^{\prime}$ is in the link of the maximal simplices of $C^{+}$. This means that $\beta^{\prime \prime} \subseteq\{1, \ldots, n\}$ is not a face of $\Delta^{\prime}$ exactly when either $T^{-} \subseteq \beta^{\prime \prime}$ or $\beta^{\prime \prime}$ is not a face of $\Delta$ and $\beta^{\prime \prime} \neq T^{+} \cup \tau \cup \gamma^{\prime}$ for any $\tau \subsetneq T^{-}$and $\gamma^{\prime}$ in the link of the maximal simplices of $C^{+}$. This proves the claim.

We now show that $\operatorname{rad}\left(I_{\text {flip }}\right) \subseteq M$. Let $x^{\alpha^{\prime \prime}}$ be a minimal generator of $I_{\text {flip }}$ such that $x^{\operatorname{supp}\left(\alpha^{\prime \prime}\right)}$ is a minimal generator of $\operatorname{rad}\left(I_{\text {flip }}\right)$. If $x^{\alpha^{\prime \prime}}$ is also a minimal generator of $I$, then $x^{\operatorname{supp}\left(\alpha^{\prime \prime}\right)}$ is in the square free ideal generated by all the generators of $\operatorname{rad}(I)$ except $x^{\operatorname{supp}(\alpha)}$, so $x^{\operatorname{supp}\left(\alpha^{\prime \prime}\right)} \in M$. Since $x^{\operatorname{supp}(\beta)} \in M$, the only case left to consider is $\alpha^{\prime \prime}=\alpha+g$ for some $g \neq 0$ with $\beta \not \leq g$. Write $g=\alpha^{\prime}+\beta^{\prime}+\gamma^{\prime}$, where $\operatorname{supp}\left(\alpha^{\prime}\right) \subseteq \operatorname{supp}(\alpha)$, $\operatorname{supp}\left(\beta^{\prime}\right) \subsetneq \operatorname{supp}(\beta)$, and $\operatorname{supp}\left(\gamma^{\prime}\right) \cap T=\emptyset$. Choose $\delta^{\prime}$ so that $\delta^{\prime}+\alpha^{\prime}=l \alpha+\tilde{\alpha}$ for some $l \geq 0$, where $\operatorname{supp}(\tilde{\alpha})=T^{+} \backslash\{p\}$ for some $p \in T^{+}$. Since $x^{\alpha^{\prime \prime}}$ is a minimal 
generator of $I_{\text {flip }}$ different from $x^{\alpha}$, it is in $W_{\alpha-\beta}$. It thus follows that $x^{\alpha+g+\delta^{\prime}} \in W_{\alpha-\beta}$, and so, because $x^{\alpha}-x^{\beta} \in W_{\alpha-\beta}$, we have $x^{(l+1) \beta+\tilde{\alpha}+\beta^{\prime}+\gamma^{\prime}} \in W_{\alpha-\beta}$ and thus in $I$. Since $\operatorname{supp}\left((l+1) \beta+\tilde{\alpha}+\beta^{\prime}\right)=T \backslash\{p\}, x^{(T \backslash\{p\}) \cup \operatorname{supp}\left(\gamma^{\prime}\right)} \in \operatorname{rad}(I)$ and thus $\operatorname{supp}\left(\gamma^{\prime}\right)$ is not in the link of the maximal simplices of $C^{+}$. Because $\operatorname{supp}\left(\gamma^{\prime}\right)=\operatorname{supp}\left(\alpha^{\prime \prime}\right) \backslash T$, this means $x^{\alpha^{\prime \prime}} \in M$, and therefore $\operatorname{rad}\left(I_{\text {flip }}\right) \subseteq M$. Now because $\Delta\left(\operatorname{rad}\left(I_{\text {flip }}\right)\right)$ and $\Delta^{\prime}$ are both triangulations of $\mathcal{A}$, this inclusion cannot be proper. So $M=\operatorname{rad}\left(I_{\text {flip }}\right)$, and thus $\Delta\left(\operatorname{rad}\left(I_{\text {flip }}\right)\right)$ is the result of performing a bistellar flip on $\Delta(\operatorname{rad}(I))$.

Theorem 5.2 gives a map from the flip graph of monomial $A$-graded ideals and their flips to the graph of triangulations of $\mathcal{A}$ and their bistellar flips. Peeva [22, Theorem 10.13] has shown that this map is not always surjective. There is, however, one special case where there is an isomorphism between the two graphs. Recall that an integer matrix $A$ is unimodular if all maximal minors of $A$ have the same absolute value. A triangulation of $\mathcal{A}$ is unimodular if all simplices have normalized volume one [22, Section 8].

Theorem 5.5. If $\Delta$ is a unimodular triangulation of $\mathcal{A}$, then the Stanley-Reisner ideal $I(\Delta)$ is A-graded. If A is unimodular, then its flip graph is isomorphic to its Baues graph.

Proof. The first claim is what is actually proved in Lemma 10.14 of [22], although the statement is weaker there. The second claim is an immediate consequence as, if $I(\Delta)$ is $A$-graded, it must be the only $A$-graded ideal with that radical.

We note that this means that a unimodular triangulation with no flips gives rise to a disconnected toric Hilbert scheme. This is true even if the whole configuration is not unimodular.

\section{Toward a Disconnected Toric Hilbert Scheme}

We conclude with some results that suggest the existence of a disconnected toric Hilbert scheme. As mentioned earlier, Santos [18] has recently constructed a six-dimensional integral point configuration with 324 points for which there is a triangulation that admits no bistellar flips. Hence this configuration has a disconnected Baues graph. By the results in [21] and the previous section, every monomial $A$-graded ideal $I$ is supported on a triangulation of $\mathcal{A}$ via the correspondence $I \mapsto \Delta(\operatorname{rad}(I))$, and if two monomial $A$-graded ideals are adjacent in the flip graph of $A$, then either they are supported on the same triangulation or on two triangulations that are adjacent in the Baues graph of $A$. Just as for monomial $A$-graded ideals, there is a notion of coherence for triangulations of $\mathcal{A}$. Every coherent triangulation of $\mathcal{A}$ (often called a regular triangulation in the literature) supports at least one monomial $A$-graded ideal, and at least one of these ideals is coherent (see Chapter 8 in [22]). On the other hand, Peeva has shown that if a triangulation of $\mathcal{A}$ is non-coherent/non-regular, then there may be no monomial $A$-graded ideal whose radical is the Stanley-Reisner ideal of this triangulation (see 
Theorem 10.13 in [22]). Hence in order for Santos's example to lift to an example of a disconnected toric Hilbert scheme, it suffices to show that there is a monomial $A$ graded ideal whose radical is the Stanley-Reisner ideal of his isolated (non-regular) triangulation. A straightforward search for such a monomial $A$-graded ideal from his $6 \times 324$ matrix is, however, a daunting computational endeavor. Nonetheless, Santos's disconnected Baues graph seems to be evidence in favor of a disconnected flip graph.

Recall that every coherent monomial $A$-graded ideal has at least $n-d$ neighbors in the flip graph of $A$. We say that a monomial $A$-graded ideal is flip deficient if its valency in the flip graph of $A$ is strictly less than $n-d$. All flip deficient monomial $A$-graded ideals are necessarily non-coherent. Before Santos constructed an isolated triangulation, several examples of flip deficient triangulations (triangulations with valency less that $n-d$ in the Baues graph) were produced as evidence in support of the existence of a disconnected Baues graph. The first such examples appear in [4]. We provide examples of flip deficient monomial $A$-graded ideals.

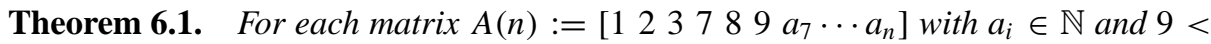
$a_{7}<\cdots<a_{n}$, there is a monomial $A(n)$-graded ideal with at most $n-3<n-1=$ $\operatorname{corank}(A(n))$ flips.

Proof. For the matrix $A=\left[\begin{array}{lllll}1 & 2 & 3 & 7 & 8\end{array}\right.$ 9 $]$, the monomial ideal $J=\left\langle x_{1} x_{5}, x_{2} x_{4}, x_{1} x_{4}\right.$, $x_{1} x_{2}, x_{4} x_{6}, x_{2} x_{6}, x_{1} x_{6}, x_{3} x_{4}, x_{2}^{2} x_{3}, x_{1} x_{3}, x_{2} x_{5}^{2}, x_{2}^{2} x_{5}, x_{1}^{2}, x_{3}^{2}, x_{2}^{4}, x_{3} x_{5}^{3}, x_{4}^{2} x_{5}^{2}, x_{4}^{3}, x_{5}^{6}, x_{4}$ $\left.x_{5}^{4}\right\rangle$ is $A$-graded. The flippable binomials of $J$ are $x_{5}^{6}-x_{3} x_{6}^{5}, x_{2} x_{6}-x_{3} x_{5}$ and $x_{3}^{2}-x_{2}^{3}$. In this example, there are 2910 monomial $A$-graded ideals in total and the flip graph of $A$ is connected.

Consider the monomial ideal $J^{\prime}=J+\left\langle x_{7}, \ldots, x_{n}\right\rangle \subseteq k\left[x_{1}, \ldots, x_{n}\right]$ and a degree $b \in \mathbb{N} A(n)=\mathbb{N} A=\mathbb{N}$. All the monomials in $k\left[x_{1}, \ldots, x_{n}\right]$ of $A(n)$-degree $b$ that are divisible by at least one of $x_{7}, \ldots, x_{n}$ are in $J^{\prime}$ by construction. Among the monomials in $k\left[x_{1}, \ldots, x_{6}\right]$ of degree $b$ (there is at least one such since $b \in \mathbb{N} A$ ), there is precisely one that is not in $J$ and hence not in $J^{\prime}$ and hence $J^{\prime}$ is $A(n)$-graded. If $x^{\alpha}-x^{\beta} \in$ $k\left[x_{1}, \ldots, x_{6}\right]$ is flippable for $J^{\prime}$, then $\operatorname{in}_{x^{\alpha} \succ x^{\beta}}\left(\left\langle x^{\alpha}-x^{\beta}\right\rangle+\left\langle x^{\gamma}: x^{\gamma}\right.\right.$ minimal generator of $\left.J, \gamma \neq \alpha\rangle+\left\langle x_{7}, \ldots, x_{n}\right\rangle\right)=J^{\prime}$. The only non-trivial $S$-pairs that are produced during this calculation are those between $x^{\alpha}-x^{\beta}$ and a monomial minimal generator $x^{\gamma}$ of $J$. Since the resulting initial ideal equals $J^{\prime}$, it follows that $\operatorname{in}_{x^{\alpha} \succ x^{\beta}}\left(\left\langle x^{\alpha}-x^{\beta}\right\rangle+\left\langle x^{\gamma}: x^{\gamma}\right.\right.$ minimal generator of $J, \gamma \neq \alpha\rangle)=J$ and hence $x^{\alpha}-x^{\beta}$ is flippable for $J$. So $x^{\alpha}-x^{\beta}$ must be one of the three flippable binomials of $J$. Additionally, each of the minimal generators $x_{7}, \ldots, x_{n}$ of $J^{\prime}$ provides a flippable binomial and hence $J^{\prime}$ has $3+(n-6)=n-3$ flippable binomials.

Remark 6.2. We have not found matrices of corank three with flip deficiency in our experiments. However, flip deficiency occurs in corank four. Consider $A=\left[\begin{array}{lllll}3 & 6 & 8 & 10 & 15\end{array}\right]$ and its monomial $A$-graded ideal

$$
\left\langle a e, b d, a b^{2}, b e, a^{2}, d^{2}, e^{2}, b^{3}, a b c^{2}\right\rangle
$$


The neighboring monomial $A$-graded ideals are:

$$
\begin{aligned}
& \left\langle a e, b d, a b^{2}, b e, d e, a^{2}, d^{2}, e^{2}, b^{3}\right\rangle \text { from } d e-a b c^{2}, \\
& \left\langle a e, b d, a b^{2}, b^{2} e, a^{2}, d^{2}, e^{2}, b^{3}, a c d, a b c^{2}\right\rangle \text { from } a c d-b e, \text { and } \\
& \left\langle a e, b d, a b^{2}, a d^{2}, b e, b^{2} c, a^{2}, d^{3}, e^{2}, b^{3}, a b c^{2}, d^{2} e\right\rangle \text { from } b^{2} c-d^{2} .
\end{aligned}
$$

Remark 6.3. It is interesting to note that flip-deficient triangulations do not exist for $d \leq 3$ while there are flip-deficient monomial $A$-graded ideals even when $d=1$.

Proposition 6.4. There are flip-deficient monomial A-graded ideals that are squarefree (i.e., supported on unimodular triangulations).

Proof. This result was pointed out to us by one of the referees and is based on Remark 3.2 in [4]. Let $\mathcal{A}$ be the vertices of the cube-octahedron of volume $48-8=40$ obtained by cutting the eight corners of the unit cube $[-1,1]^{3}$ plus the origin. Then the flip-deficient triangulation of $\mathcal{A}$ from Remark 3.2 in [4] has 20 tetrahedra, each of volume two. The points in $\mathcal{A}$ lie in the sublattice $\left\{(x, y, z) \in \mathbb{Z}^{3}: x+y+z \in 2 \mathbb{Z}\right\}$. Any affine transformation that sends this lattice bijectively to $\mathbb{Z}^{3}$ makes the triangulation unimodular. The Stanley-Reisner ideal $I$ of this unimodular triangulation $\Delta$ is $A$-graded (Lemma 10.14 in [22]). Since a unimodular triangulation supports exactly one monomial $A$-graded ideal, no flip of $I$ leads to another monomial $A$-graded ideal supported on $\Delta$. Further, no two flips of $I$ can lead to monomial $A$-graded ideals supported on the same neighboring triangulation of $\Delta$ in the Baues graph of $A$ since all flips of $I$ are supported on distinct circuits of $\mathcal{A}$ which yield distinct neighboring triangulations of $\Delta$.

Remark 6.5. The above computations were made using two different programs. Starting with a monomial initial ideal of the toric ideal $I_{A}$ one can compute all monomial $A$-graded ideals in the same connected component as this initial ideal by using the results in Section 2 to calculate all the neighbors of a monomial $A$-graded ideal. This computation can be done using the program TiGERS [11] with the command tigers -iAe filename where filename is the standard input file for TiGERS with the data of the matrix $A$. In order to find all monomial $A$-graded ideals, we resort to a second program (available from the authors) that first computes the Graver basis of $A$ and then systematically constructs weakly $A$-graded monomial ideals by choosing one monomial from each Graver binomial to be in the ideal (see Lemma 2.6). The program then compares the Hilbert series of each such ideal against that of an initial ideal of $I_{A}$ to decide if it is A-graded. Comparing the total number of ideals produced by the two programs gives a convenient way to decide if the flip graph is connected. These algorithms are discussed further in [20].

We conclude with an algorithmic issue concerning the enumeration of all $A$-graded monomial ideals in the same connected component as a fixed one. The main program in TiGERS enumerates the vertices of the state polytope of $I_{A}$ by using the reverse search strategy of Avis and Fukuda [2], which requires only the current vertex to be stored at any given time. The input to the program is any one monomial initial ideal of $I_{A}$ from which the program reconstructs all the others without needing to consult the list of ideals it has 
already found. An essential requirement of this algorithm is a method by which the input ideal can be distinguished from any other monomial initial ideal of $I_{A}$ by considering only the edges of the state polytope. This is done in TiGERS as follows:

Suppose $M_{1}$ and $M_{2}$ are two monomial initial ideals of $I_{A}$ induced by the weight vectors $w_{1}$ and $w_{2}$, respectively. Let $\mathcal{G}_{1}$ and $\mathcal{G}_{2}$ be the corresponding reduced Gröbner bases of $I_{A}$. Then for each facet binomial $x^{a}-x^{b}$ in $\mathcal{G}_{1}$ we have $w_{1} \cdot(a-b)>0$ and for each facet binomial $x^{\alpha}-x^{\beta} \in \mathcal{G}_{2}$ we have $w_{2} \cdot(\alpha-\beta)>0$. The reduced Gröbner bases $\mathcal{G}_{1}$ and $\mathcal{G}_{2}$ coincide if and only if each facet binomial $x^{\alpha}-x^{\beta}$ of $\mathcal{G}_{2}$ satisfies the inequality $w_{1} \cdot(\alpha-\beta)>0$. Suppose the input is a fixed initial ideal of $I_{A}$. By the previous observation, every other monomial initial ideal of $I_{A}$ will have a mismarked facet binomial with respect to the term order inducing the input initial ideal and hence can be distinguished from it. The following example shows that monomial $A$-graded ideals cannot be distinguished by checking the orientation of their flippable binomials.

Example 6.6. Consider $A=\left[\begin{array}{lllll}3 & 4 & 5 & 13 & 14\end{array}\right]$ and its non-coherent monomial $A$-graded ideal

$$
M=\left\langle c d^{5}, c^{2} e^{3}, b e, d^{9}, b^{2}, c^{3}, a^{6}, b d, a e^{2}, a d^{3}, a c^{2}, a^{2} d, a^{2} b, b c, a^{3} e, a^{3} c\right\rangle .
$$

The flippable binomials of $M$ are $a e^{2}-c d^{2}, c^{3}-a^{5}$, and $d^{9}-c e^{8}$. With respect to the weight vector $w=(0,0,1,20,22)$, each of these flippable binomials has its positive term as the leading term and hence $M$ cannot be distinguished from $\operatorname{in}_{w}\left(I_{A}\right)$ by checking whether its flippable binomials are mismarked with respect to $w$.

\section{Acknowledgments}

We thank Bernd Sturmfels for helpful conversations. We also thank the referees for several useful comments. In particular Proposition 6.4 was pointed out to us by one of the referees.

\section{References}

1. V. I. Arnold. A-graded algebras and continued fractions. Comm. Pure Appl. Math., 42(7):993-1000, 1989.

2. D. Avis and K. Fukuda. A basis enumeration algorithm for convex hulls and vertex enumeration of arrangements and polyhedra. Discrete Comput. Geom., 8:295-313, 1992.

3. D. Bayer and I. Morrison. Standard bases and geometric invariant theory. I. Initial ideals and state polytopes. J. Symbolic Comput., 6(2-3):209-217, 1988.

4. J. Deloera, F. Santos, and J. Urrutia. The number of geometric bistellar neighbors of a triangulation. Discrete Comput. Geom., 21:131-142, 1999.

5. D. Eisenbud and B. Sturmfels. Binomial ideals. Duke Math. J., 84(1):1-45, 1996.

6. G. Ewald. Combinatorial Convexity and Algebraic Geometry. Springer-Verlag, New York, 1996.

7. W. Fulton. Introduction to Toric Varieties. Princeton University Press, Princeton, NJ, 1993. (The William H. Roever Lectures in Geometry.)

8. V. Gasharov and I. Peeva. Deformations of codimension 2 toric varieties. Compositio Math., 123:225-241, 2000.

9. R. Hartshorne. Connectedness of the Hilbert scheme. Publ. Math. IHES, 29:261-304, 1966.

10. S. Hoşten and D. Maclagan. The vertex ideal of a lattice. math. co/0012197. 
11. B. Huber and R. R. Thomas. Computing Gröbner fans of toric ideals. Experimental Math., 9:321-331, 2000. TiGERS available from http: //www. math. washington. edu/ thomas/programs. html.

12. E. Korkina. Classification of A-graded algebras with 3 generators. Nederl. Akad. Wetensch. Indag. Math (N.S.), 3(1):27-40, 1992.

13. E. Korkina, G. Post, and M. Roelofs. Classification of generalized A-graded algebras with 3 generators. Bull. Sci. Math., 119:267-287, 1995.

14. I. Peeva and M. Stillman. Local equations for the toric Hilbert scheme. Adv. in Appl. Math., 25:307-321, 2000 .

15. I. Peeva and M. Stillman. Toric Hilbert schemes. Duke Math. J., to appear.

16. J. Rambau and G. M. Ziegler. Projections of polytopes and the generalized Baues conjecture. Discrete Comput. Geom., 16:215-237, 1996.

17. V. Reiner. The generalised Baues problem. In L. Billera, A. Björner, C. Greene, R. Simion, and R. Stanley, editors, New Perspectives in Algebraic Combinatorics, pages 293-336. Cambridge University Press, Cambridge, 1999.

18. F. Santos. A point configuration whose space of triangulations is disconnected. J. Amer. Math. Soc., 13:611-637, 2000.

19. R. P. Stanley. Combinatorics and Commutative Algebra, second edition. Birkhäuser, Boston, MA, 1996.

20. M. Stillman, B. Sturmfels, and R. Thomas. Algorithms for the toric Hilbert scheme. D. Eisenbud, D. Grayson, M. Stillman and B. Sturmfels, editors, Computations in Algebraic Geometry using Macaulay 2, to appear.

21. B. Sturmfels. The geometry of $A$-graded algebras. alg-geom/9410032.

22. B. Sturmfels. Gröbner Bases and Convex Polytopes. American Mathematical Society, Providence, RI, 1996.

23. B. Sturmfels and R. R. Thomas. Variation of cost functions in integer programming. Math. Programming, 77(3, Ser. A):357-387, 1997.

Received May 15, 2000, and in revised form March 8, 2001. Online publication January 7, 2002. 